\title{
Asymptotic Analysis on Secrecy Capacity in Large-Scale Wireless Networks
}

\author{
Jinbei Zhang, Luoyi Fu, Xinbing Wang \\ Dept. of Electronic Engineering \\ Shanghai Jiao Tong University, China \\ Email: \{abelchina, yiluofu, xwang8\}@ sjtu.edu.cn
}

\begin{abstract}
Since wireless channel is vulnerable to eavesdroppers, the secrecy during message delivery is a major concern in many applications such as commercial, governmental and military networks. This paper investigates information-theoretic secrecy in large-scale networks and studies how capacity is affected by the secrecy constraint where the locations and channel state information (CSI) of eavesdroppers are both unknown. We consider two scenarios: 1) non-colluding case where eavesdroppers can only decode messages individually; and 2) colluding case where eavesdroppers can collude to decode a message. For the non-colluding case, we show that the network secrecy capacity is not affected in order-sense by the presence of eavesdroppers. For the colluding case, the per-node secrecy capacity of $\Theta\left(\frac{1}{\sqrt{n}}\right)$ can be achieved when the eavesdropper density $\psi_{e}(n)$ is $O\left(n^{-\beta}\right)$, for any constant $\beta>0$ and decreases monotonously as the density of eavesdroppers increases. The upper bounds on network secrecy capacity are derived for both cases and shown to be achievable by our scheme when $\psi_{e}(n)=O\left(n^{-\beta}\right)$ or $\psi_{e}(n)=\Omega\left(\log \frac{\alpha-2}{\alpha} n\right)$, where $\alpha$ is the path loss gain. We show that there is a clear tradeoff between the security constraints and the achievable capacity. Furthermore, we also investigate the impact of secrecy constraint on the capacity of dense network, the impact of active attacks and other traffic patterns as well as mobility models in the context.
\end{abstract}

\section{INTRODUCTION}

Although facilitating communications through quick deployment and low cost, the broadcast nature of wireless channel makes it vulnerable to attacks such as eavesdropping and jamming, which are important concerns for commercial, governmental and military networks. Traditional solutions are based on cryptographic methods such as the well-known RSA public key cryptosystem. However, due to the expensive key distribution, the rapid growth of computation power and improvement on decoding technology, cryptographic techniques encounter some limitations, especially as the network size increases. Hence, to avoid such limitations, this paper focuses on information theoretic security where eavesdroppers are assumed to have infinite computational power.

The basis for information theoretic security stems from Shannon's notion of perfect secrecy [1], which is then extended to noisy channels by Wyner [2] and later by Csiszár and Körner [3]. Information theoretic security is achieved by exploiting the difference between channels of legitimate

An earlier version of this paper appeared in the Proceedings of IEEE Infocom 2012(mini) [15]. nodes and that of eavesdroppers, which requires the intended receiver to have a stronger channel than eavesdroppers. Recently, securing wireless communications at the physical layer is intriguing renewed interests among research area. Haenggi [4] and Pinto et al. [5] study the in-degree and outdegree distributions under the security constraints. As is shown in both papers, even a small number of eavesdroppers will cause dramatic decreasing in nodes' connectivity. To guarantee the secret transmission, Geol and Negi [6] propose artificial noise generation to suppress eavesdroppers' receiving signal. The independence of fading channels is exploited to generate noise to suppress eavesdroppers' channels taking advantage of cooperative schemes [7] and multiple antennas [8], [9]. Furthermore, Barros et al. [10] show that theoretic information secrecy can be achieved by fading alone if channel state information (CSI) is available.

However, so far the research about information theoretic security mainly focuses on distinctive techniques to enhance the security, yet little is known about their impact on network performance such as capacity, delay, etc, especially in large scale wireless networks. As some exceptions, Vasudevan et al. [12] study the secrecy capacity issue in a large-scale network. Specifically, they introduce helper nodes around transmitters to generate noise to degrade eavesdroppers' channel and utilize channel fading gain of receivers to enhance secure communications. The impact of secrecy guard zone on capacity is investigated by Koyluoglu et al. [13] and Zhou et al. [14]. On the other hand, what is the upper bound of secrecy capacity is unknown. Furthermore, some of pre-known information is needed in the previous works, such as pre-known CSI information of receivers or some pre-known location information of eavesdroppers. These pre-known information can be used by transmitters to differentiate receivers' channels from eavesdroppers'. And, in real applications it is difficult to obtain such information a prior, especially in large scale wireless networks. Therefore, a fundamental question arises: what will be the performance of secrecy capacity, if both the CSI and location information are unknown to legitimate nodes?

We are thus motivated to investigate this issue in static wireless networks. Our main idea to solve the aforementioned problem is to let a receiver distinguish its own channel by adopting self-interference cancelation. More precisely, we assume each receiver is equipped with three antennas, one for message reception and the other two for simultaneous artificial noise generation to suppress eavesdroppers' channels. Since 
the three antennas are all equipped on one node, the noise generated by the receiver itself can be eliminated through the technique of antenna cancelation proposed in [17]. This differs our noise generation pattern from previous works and we will show in later part that such difference can dramatically improve network secrecy capacity.

Our main contributions are summarized as follows:

- In the non-colluding case, the optimal per-node secrecy capacity $\Theta\left(\frac{1}{\sqrt{n}}\right)$ is achievable in the presence of eavesdroppers. This result holds even in the scenario where there are more eavesdroppers than legitimate nodes in the network.

- In the colluding case, we establish the relationship between the secrecy capacity and the tolerable number of eavesdroppers. More importantly, we first derive the upper bound for secrecy capacity which is achievable.

- We identify the underlying interference model to capture the fundamental impact of secrecy constraints. This model relies weakly on the specific settings such as traffic pattern and mobility models of legitimate nodes. Hence, our study can be flexibly applied to more general cases and shed insights into the design and analysis of future wireless networks.

The rest of this paper is organized as follows. In Section II, we present the system model. Asymptotic analysis on different scenarios is carried out in Section III and IV. We investigate the effect of dense network in Section V. Jamming as a different kind of network attacking is investigated in Section VI. Discussions and concluding remarks are given in Section VII and VIII, respectively.

\section{A. Related Works}

Asymptotic analysis can provide fundamental insight on network performance as the network size increases. The ground-breaking work is initiated by Gupta and Kumar [18], who study capacity performance in a network with $n$ randomly distributed nodes. They show that the per-node capacity is lower bounded by $\Omega\left(\frac{1}{\sqrt{n \log n}}\right)$ and upper bounded by $O\left(\frac{1}{\sqrt{n}}\right)$. This gap is closed later by Franceschetti et al. [20] using percolation theory. The fundamental difference behind these two works is the underlying connectivity. The communication range in [18] should be $\Theta\left(\sqrt{\frac{\log n}{n}}\right)$ to guarantee full connectivity whereas it only needs to be $\Theta\left(\sqrt{\frac{1}{n}}\right)$ in [20] to guarantee partial connectivity at the bottleneck. Later on, Grossglauser and Tse [23] further indicate the capacity can be improved to $\Theta(1)$ when mobility is introduced to nodes, at the expense of increased delay [19]. Since then, asymptotic analysis has drawn considerable attention in research area and we will give a brief introduction in the following.

While interference always has an negative effect on wireless communication, MIMO technology turns it into useful signal and hence greatly enhance the communication. In [28], Özgür et al. propose an iterative MIMO scheme to obtain a constant per-node capacity which is a great improvement compared to hop-by-hop transmissions. Infrastructure is another effective way to overcome the interference. Liu et al. [27] prove that the gain on capacity can increase linearly with the number of base station under certain circumstances. Multicast is a common traffic pattern in real networks which makes the analysis much more difficult. Li [24] proposes a tree-based routing scheme to deal with it. More recently, Wang et al. [25] and Tang et al. [26] study multicast capacity in hybrid networks. Mobility pattern plays an important part in wireless networks and Neely [19] uses queuing theory to study the delay and capacity. $\mathrm{Hu}$ [11] further study the multicast capacity in mobile large scale networks. Brownian motion is an important mobility pattern which is studied in [30] by Lin et al. . Homogeneous mobility pattern and uniform node density is the first step for the study on mobile networks. Garetto et al. [21] investigate the heterogeneous cases which include a large body of mobility models.

\section{Network Models And Definitions}

In this paper, we consider a static ad hoc network in an extended network $\mathscr{B}=[0, \sqrt{n}] \times[0, \sqrt{n}]$.

Legitimate Nodes: Legitimate nodes follow a Poisson distribution with unit intensity over the whole network. And transmitter-receiver pairs are randomly chosen such that each node is the destination of exactly one source. We denote $\mathcal{T}$ and $\mathcal{R}$ as the subsets of nodes simultaneously transmitting and receiving at a given time-slot. We assume that each legitimate node is equipped with three antennas. When a legitimate node acts as a receiver, one antenna is used for message reception while the other two are devoted to simultaneous artificial noise generation to suppress eavesdroppers' channels. The distances between the receive antenna and the two respective transmit antennas should satisfy a difference of half the wavelength. The interference can therefore be eliminated using the technique of self-interference cancelation proposed in [17]

Eavesdroppers: Independently of legitimate nodes, eavesdroppers also follow a Poisson distribution in the network with intensity $\lambda_{e}$. Let $\mathcal{E}$ be the set of eavesdroppers. We assume eavesdroppers always keep silent since they will be easily detected if active. In order to have an insight on the fundamental information theoretical secrecy capacity, we assume eavesdroppers have infinite computation ability which means that traditional cryptography method can not be applied here. We also assume that both CSI and location information of eavesdroppers are unknown to legitimate nodes.

The Physical Model: For simplicity, we denote uniform transmission power as $P_{t}$ and uniform noise generation power as $P_{r}$. The path loss between node $i$ and node $j$ is denoted by $l\left(x_{i}, x_{j}\right)$, which can be expressed as $l\left(x_{i}, x_{j}\right)=\min \left(1, d_{i j}^{-\alpha}\right)$. Here $d_{i j}$ is the transmission distance and the loss exponent $\alpha>2$. When node $i$ is transmitting messages to node $j$, the signal to interference and noise ratio (SINR) received by node $j$ over a channel of unit bandwidth can be given by:

$$
\operatorname{SINR}_{i j}=\frac{P_{t} l\left(x_{i}, x_{j}\right)}{N_{0}+\sum_{k \in \mathcal{T} \backslash\{i\}} P_{t} l\left(x_{k}, x_{j}\right)+\sum_{k \in \mathcal{R} \backslash\{j\}} P_{r} l\left(x_{k}, x_{j}\right)},
$$

where $N_{0}$ denotes the ambient noise power at the receiver. 
The SINR received by eavesdropper $e$ can be represented by: $\operatorname{SINR}_{i e}=\frac{P_{t} l\left(x_{i}, x_{e}\right)}{N_{0}+\sum_{k \in \mathcal{T} \backslash\{i\}} P_{t} l\left(x_{k}, x_{e}\right)+\sum_{k \in \mathcal{R}} P_{r} l\left(x_{k}, x_{e}\right)}$.

Secrecy Throughput Per Hop: As is defined in [3], the secure throughput between any active transmitter-receiver pair is:

$$
R_{i j}^{s}=R_{i j}-\overline{R_{i e}}=\log _{2}\left(1+\operatorname{SINR}_{i j}\right)-\log _{2}\left(1+\overline{\operatorname{SINR}_{i e}}\right)
$$

where $\overline{\operatorname{SINR}_{i e}}=\max _{e \in \mathcal{E}} \operatorname{SINR}_{i e}$.

Asymptotic Capacity: Asymptotic per node capacity $\lambda(n)$ is said to be achievable if there is a scheduling and routing scheme such that every node can transmit $\lambda(n)$ bits per second on average to its destination in the long term.

Knuth Notations: Denote $\lambda(n)=O(f(n))$ if there is a positive constant $c_{1}$ such that $\lim _{n \rightarrow \infty} P\left(\frac{\lambda(n)}{f(n)} \leq c_{1}\right)=1$ and $\lambda(n)=\Omega(f(n))$ if $f(n)=O(\lambda(n)) . \lambda(n)$ is said to be $\Theta(f(n))$ if both $\lambda(n)=O(f(n))$ and $\lambda(n)=\Omega(f(n))$ hold.

In Table 1 , we list the parameters that will be frequently used in later analysis, proofs and discussions.

\section{TABLE I: Notations}

\begin{tabular}{c|l}
\hline Notation & \multicolumn{1}{|c}{ Definition } \\
\hline \hline$n$ & The total number of legitimate nodes in the network. \\
\hline$\lambda_{s}(n)$ & The per-node secrecy capacity. \\
\hline$\psi_{e}(n)$ & $\begin{array}{l}\text { The expected density of poisson distributed eaves- } \\
\text { droppers. }\end{array}$ \\
\hline$P_{t}$ & The power to transmit packets. \\
\hline$P_{r}$ & The power to generate noise. \\
\hline$R(d)$ & $\begin{array}{l}\text { The rate that a transmitter can transmit to an intend- } \\
\text { ed receiver which is located } d \text { distance away. }\end{array}$ \\
\hline$R_{e}$ & $\begin{array}{l}\text { The rate that an eavesdropper can obtain from a } \\
\text { transmitting node. }\end{array}$ \\
\hline$R_{s}(d)$ & $\begin{array}{l}\text { The rate that a transmitter can securely transmit to an } \\
\text { intended receiver which is located } d \text { distance away. }\end{array}$ \\
\hline
\end{tabular}

\section{SECURITY CAPACITY FOR INDEPENDENT EAVESDROPPERS CASE}

In this section, we investigate secrecy capacity for independent eavesdroppers. We use percolation theory to construct the routing scheme which contains three phases, e.g., draining phase, highway transmission and delivery phase. Since our scheme should guarantee the secrecy communication, it seems that the capacity should be degraded. However, we show that regardless of the fact that the capacity will be sacrificed to ensure secrecy in draining phase and delivery phase, the bottleneck still lies in the highway phase where the secrecy capacity remains the same as that in the network without eavesdroppers.

We present the following lemma which will be quoted throughout this paper.

Lemma 1: When a legitimate node $t$ is transmitting to a legitimate receiver $r$, the maximum rate that an independent eavesdropper $e$ can obtain is upper-bounded by

$$
R_{e} \leq \min \left(\frac{P_{t} d_{t e}^{-\alpha}}{N_{0}}, \frac{P_{t}}{P_{r}}\left(1+d_{t r}\right)^{\alpha}\right)
$$

where $d_{t r}$ is the Euclidean distance between legitimate node $t$ and node $r$ and $d_{t e}$ is the distance between legitimate node $t$ and eavesdropper $e$.

Proof: First we prove the maximum SINR that eavesdroppers can obtain is $\frac{P_{t}}{P_{r}}\left(1+d_{r t}\right)^{\alpha}$. Consider the following four cases.

Case 1: When $d_{t e}$ and $d_{r e}$ are both greater than 1 , then

$$
\begin{aligned}
\operatorname{SINR}_{e} & =\frac{P_{t} l\left(x_{t}, x_{e}\right)}{N_{0}+\sum_{k \in \mathcal{T} \backslash\{t\}} P_{t} l\left(x_{k}, x_{e}\right)+\sum_{k \in \mathcal{R}} P_{r} l\left(x_{k}, x_{e}\right)} \\
& <\frac{P_{t} l\left(x_{t}, x_{e}\right)}{P_{r} l\left(x_{r}, x_{e}\right)}=\frac{P_{t} d_{t e}^{-\alpha}}{P_{r} d_{r e}^{-\alpha}} \\
& \leq \frac{P_{t} d_{t e}^{-\alpha}}{P_{r}\left(d_{r t}+d_{t e}\right)^{-\alpha}} \\
& =\frac{P_{t}}{P_{r}}\left(1+\frac{d_{r t}}{d_{t e}}\right)^{\alpha} \leq \frac{P_{t}}{P_{r}}\left(1+d_{r t}\right)^{\alpha} .
\end{aligned}
$$

Case 2: When $d_{t e}>1$ and $d_{r e}<=1$, it is obvious to see that eavesdroppers's interference is more severe than that in case 1 while the signal received is not stronger than that in case 1 . So the bound still holds.

Case 3: When $d_{t e}<=1$ and $d_{r e}>1$, we can easily see that the path loss gain of T-E pair is 1 when the bound derived in case 1 holds, which means that the bound cannot be broken by condition $d_{t e}<=1$.

Case 4: When $d_{t e}<=1$ and $d_{r e}<=1$, the SINR at eavesdroppers will not be greater than that in case 3 since eavesdroppers in case 4 suffer more interference. Hence the bound still holds.

Notice that $\operatorname{SINR}_{e}$ is also smaller than $\frac{P_{t} d_{t e}^{-\alpha}}{N_{0}}$. Hence, since $R_{e}=\log \left(1+\max \left(\operatorname{SINR}_{e}\right)\right)$, it is straightforward to conclude this lemma.

From the analysis, we can see that the rate is tight in order sense when $d_{t r}=\Theta(1)$. And according to our following analysis, the bottleneck of secrecy capacity lies in the highway phase where $d_{t r}=\Theta(1)$. Hence, from this point of view, this rate is tight.

\section{A. The Highway System}

The network is divided into non-overlapping cells with side length of $c$, where $c$ is a constant. We say that a cell is open if there is at least one node in it. Hence cells are open with probability $p=1-e^{-c^{2}}$ independently.

For ease of exposition, denote $m$ as $\sqrt{n} / \sqrt{2} c$ and we assume $m$ to be an integer, which will not change our results in order sense. As is shown in [20], when the constant $c$ is large enough, there are a lot of crossing paths in the network which behave almost as straight lines. For any $\kappa>0$, partition the network into rectangles of size $m \times\left(\kappa \log m-\epsilon_{m}\right)$ and choose $\epsilon_{m}=o(1)$ as the smallest value such that the side length is an integer. Denote $R_{i}$ as the $i$ th rectangle and $C_{i}$ as the number of edge-disjoint crossings of $R_{i}$. Then the minimal number of disjoint crossing paths $N_{p}=\min _{i} C_{i}$ can be upper bounded by $\delta \log m$ when $m$ goes to infinity and $\delta$ is a constant. Further, to make sure that there are at least as many paths as slices inside each rectangle, each rectangle is sliced into horizontal strips with constant $w=\kappa \log m / N_{p}$. 


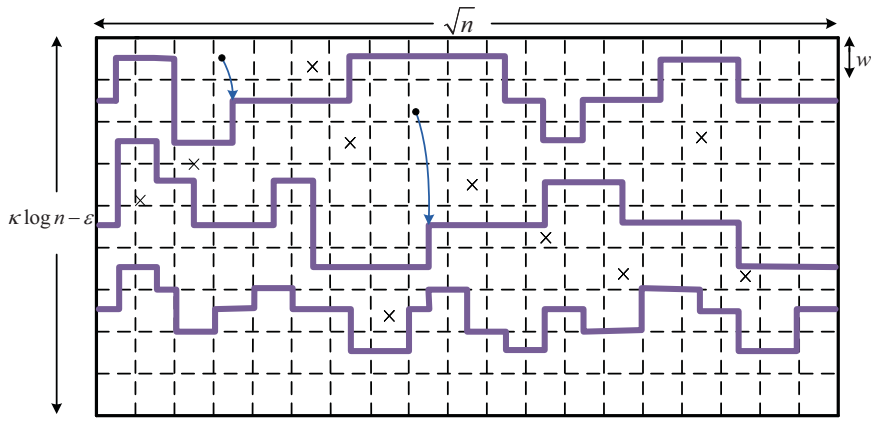

Fig. 1: There are at least $\delta \log m$ disjoint highways in each rectangle. Nodes in $i$-th slice will transmit to nodes located in the $i$-th highway and crosses denote eavesdroppers.

Our packet routing scheme includes three steps:

Step 1: Each source in the $i$-th slice transmits directly to a legitimate relay located on the $i$-th path. The relay is chosen in a way such that it is closest to the source among all other nodes on the $i$-th path, as is shown in Fig. 1.

Step 2: Packets are relayed horizontally through the highway and then along a vertical highway until it arrives at an exit point closest to the destination in a multi-hop fashion.

Step 3: Packets are directly delivered from the highway to the destination similar to the first step.

\section{B. Analysis of Secrecy Capacity}

Next we present our scheduling scheme and compute the lower bound of the legitimate receiver's rate. Note that our scheduling scheme is different from that proposed in [20], since we should take the issue of secrecy into account. And the basic idea is to space concurrent transmission sufficiently far away so that the interference is tolerable.

Lemma 2: When a legitimate node is transmitting to a legitimate receiver which is located $d$ cells apart, the minimum rate that the legitimate node can receive is lower-bounded by $c_{2} P_{t} d^{-\alpha}$, where $c_{2}$ is a constant.

Proof: First we compute the interference at the receiver. Divide the network into disjoint subsquares of $(k+d) \times(k+$ d) cells, where $k$ will be explained later. Every cell in each subsquare takes turn to transmit. Consider a given transmitterreceiver pair, the eight closest transmitters and receivers are located at distance of at least $c k$ and $c(k+d-1)$ from the receiver. The sixteen next closest transmitters and receivers are located at distance at least $c(2 k+d)$ and $c(2 k+2 d-1)$ away from the receiver and so on. Taking into consideration all the interferences in the whole network, the interference at the intended destination can be upper-bounded as follows:

$$
\begin{aligned}
I(d) & \leq \sum_{i=1}^{\infty} 8 i\left(P_{t} l(c(i(k+d)-d))+P_{r} l(c(i(k+d)-1))\right) \\
& \leq \sum_{i=1}^{\infty} 8 i\left(P_{t}+P_{r}\right) l(c i k) \\
& =\left(P_{t}+P_{r}\right)(k c)^{-\alpha} \sum_{i=1}^{\infty} 8 i(c i)^{-\alpha} .
\end{aligned}
$$

Note that $\sum_{i=1}^{\infty} 8 i(c i)^{-\alpha}$ converges to a constant $c_{1}^{\prime}$ when $\alpha \geq$ 2 .

Since the distance from the transmitter to the designated receiver is at most $c(d+1)$, the receiving signal $S(d)$ can be lower-bounded by

$$
\begin{aligned}
S(d) & \geq P_{t} l(c(d+1)) \\
& =P_{t}(c(d+1))^{-\alpha} .
\end{aligned}
$$

Notice that $l(c(d+1))=(c(d+1))^{-\alpha}$ since $d$ is an integer and $c$ is greater than 1 .

Now the accurate rate that the legitimate receiver may achieve can be derived as follows:

$$
\begin{aligned}
R(d) & =\log \left(1+\frac{S(d)}{N_{0}+I(d)}\right) \\
& \geq \log \left(1+\frac{P_{t}(c(d+1))^{-\alpha}}{N_{0}+c_{1}^{\prime}\left(P_{t}+P_{r}\right)(k c)^{-\alpha}}\right) \\
& \geq c_{2}^{\prime} P_{t}(c(d+1))^{-\alpha} \\
& \geq c_{2} P_{t} d^{-\alpha},
\end{aligned}
$$

when choosing $k=\Theta\left(P_{r}^{\frac{1}{\alpha}}\right)$ and $c_{2}$ is a constant.

Now we will show that secrecy communication can be assured for any T-R pairs by appropriately spacing for concurrent transmissions.

Theorem 1: For any legitimate transmitter-receiver pair which is spaced at a distance of $d$ cells apart, there exists an $R_{s}(d)=\Omega\left(d^{-\alpha-4}\right)$, so that the receiver can receive at a rate of $R_{s}(d)$ securely from the transmitter.

Proof: According to the definition of secure rate and combining with Lemma 1 and Lemma 2, the secrecy rate $R_{s}(d)$ each cell can transmit can be denoted as:

$$
\begin{aligned}
R_{s}(d) & =\frac{1}{(k+d)^{2}}\left(R(d)-R_{e}\right) \\
& \geq \frac{1}{(k+d)^{2}}\left(c_{2} P_{t} d^{-\alpha}-c_{3} \frac{P_{t}}{P_{r}} d^{\alpha}\right)
\end{aligned}
$$

where $\frac{1}{(k+d)^{2}}$ is the time utilization factor, $c_{2}$ and $c_{3}$ are both constants.

Let $P_{r}=2 \frac{c_{3}}{c_{2}} d^{2 \alpha}$. Hence, to bound the interference incurred to the intended receiver, according to Equation (5), $k=\Theta\left(P_{r}^{\frac{1}{\alpha}}\right)=\Theta\left(d^{2}\right)$. Therefore, the secrecy rate each cell can receive is $\Omega\left(d^{-\alpha-4}\right)$.

Theorem 1 indicates positive secrecy rate is achievable even under the worst attack. In order to calculate per-node secrecy capacity, we first compute the number of legitimate nodes in each cell and then derive the traffic load that each node in the highway should relay, as are shown in the following two lemmas.

Lemma 3: There are at most $\log n$ legitimate nodes in each cell of constant size $c^{2}$ w.h.p. ${ }^{1}$.

${ }^{1}$ In this paper, w.h.p stands for with high probability, which means the probability tends to 1 as $n$ goes to infinity. 
Proof: Let $A_{i}$ be the number of legitimate nodes in cell $i$ and $A$ be the maximum number of $A_{i}$. Hence, we have

$$
\begin{aligned}
P(A \geq \log n) & =P\left(\max A_{i} \geq \log n\right) \\
& \leq P\left(\cup_{i}\left(A_{i} \geq \log n\right)\right) \\
& \leq \sum_{i} P\left(A_{i} \geq \log n\right) \\
& \leq \frac{n}{c^{2}} e^{-c^{2}}\left(\frac{c^{2} e}{\log n}\right)^{c^{2} \log n} \\
& =\frac{1}{c^{2}} e^{-c^{2}}\left(\frac{c^{2} e^{1+1 / c^{2}}}{\log n}\right)^{c^{2} \log n} \rightarrow 0 .
\end{aligned}
$$

Note that the third inequality follows from union bounds and the fourth one follows from Chernoff bounds [31].

Lemma 4: If nodes are poisson distributed with intensity $\psi(n)$ in the network $\mathscr{B}$, partition the network into disjoint regions with same size $f(n)$, let $N_{i}$ be the number of nodes inside region $i$. We have

$$
P\left(\frac{1}{2} f(n) \psi(n) \leq N_{i} \leq 2 f(n) \psi(n), \forall i\right)=1
$$

when $f(n) \psi(n) \geq \log _{4 / e} n$ and $f(n)=\Omega(1)$.

Proof: The number of nodes inside each region $N_{i}$ is a poisson variable and denote its expectation as $\psi$. Hence, we have $\psi=E\left(N_{i}\right)=f(n) \psi(n)$. Letting $\mathrm{N}$ be the maximum number of $N_{i}$, for all $i$. Under similar derivation of Lemma 3 , we can get that

$$
\begin{aligned}
P(N \geq 2 f(n) \psi(n)) & \leq \frac{n}{f(n)} P\left(N_{i} \geq 2 f(n) \psi(n)\right) \\
& \leq \frac{n}{f(n)} e^{-\psi}\left(\frac{e \psi}{2 \psi}\right)^{2 \psi} \\
& =\frac{n}{f(n)}\left(\frac{e}{4}\right)^{f(n) \psi(n)} \\
& \leq \frac{1}{f(n)} \rightarrow 0
\end{aligned}
$$

when $f(n) \psi(n) \geq \log _{4 / e} n$ and $f(n)=\Omega(1)$.

Similarly, we can show that $\min _{i} N_{i}$ is greater than $\frac{1}{2} f(n) \psi(n)$ w.h.p. when conditions hold.

Theorem 2: With $n$ legitimate nodes randomly distributed in $\mathscr{B}$, the achievable per-node secrecy throughput under the existence of independent eavesdroppers is $\Omega\left(\frac{1}{\sqrt{n}}\right)$.

Proof: As is shown in the routing scheme, the maximum distance between source and relay on the highway is no larger than $\kappa \log m+2 c$ in the first step. Applying Theorem 1, we obtain that one node in the cell can transmit securely at rate $\Omega\left(\log ^{-\alpha-4} n\right)$ to the relay. Since there may be multiple nodes inside the cell, they should share the transmission chances. The number of nodes inside each cell can be bounded as $O(\log n)$ according to Lemma 3 . Hence, the achievable secrecy capacity is $\Omega\left(\log ^{-\alpha-5} n\right)$ in the draining phase. Note that since the delivery phase is a reverse process of the draining phase, the secrecy capacity of the delivery phase is the same as that of draining phase.

In the highway phase, the transmission range between T-R pairs is at most $2 \sqrt{2} c$. Hence each node on the highway can transmit securely at rate $\Omega(1)$ to the next relay by applying
Theorem 1. According to the routing scheme, each source in the $i$-th slice transmit packets to the $i$-th highway in the same rectangle. Since the density of legitimate nodes is 1 and the size of each slice is $w \sqrt{n}$, which satisfy the conditions given by Lemma 4, we obtain that the maximum number of legitimate nodes inside each slice is no larger than $2 w \sqrt{n}$. Hence, the traffic load on each relay node in the highway is at most $2 w \sqrt{n}$ nodes. Therefore, the secrecy capacity of the highway phase is $\Omega\left(\frac{1}{\sqrt{n}}\right)$.

Based on the results above, we conclude that per-node secrecy capacity is $\Omega\left(\frac{1}{\sqrt{n}}\right)$.

\section{The Optimality of Our Scheme}

We first consider the case where no eavesdropper exists in the network. Due to the broadcast nature of wireless channel, every concurrent transmission will incure interference to other transmissions. The following lemma shows that there is a constraint on the total network throughput underlying this fundamental physical model.

Lemma 5: When $n$ nodes are identically and randomly located in a wireless network and source-destination pairs are randomly chosen, the per-node throughput $\lambda(n)$ is upper bounded by $O\left(\frac{1}{\sqrt{n}}\right)$.

Proof: Let $L$ be the expected distance between all sourcedestination pairs. Hence $L=\Theta(\sqrt{n})$ [18]. Denote $r$ and $T(r)$ as the average transmission range and rate of each hop respectively. Let $l$ be the average distance that simultaneous transmissions can occure. Since the expected number of hops that a packet should travel is $L / r$, the total traffic load the network should carry is $n \lambda(n) L / r$. And the total traffic that the network can carry is $\frac{n}{l^{2}} T(r)$. Hence, we obtain that $n \lambda(n) L / r \leq \frac{n}{l^{2}} T(r)$, which means $\lambda(n) \leq \frac{r T(r)}{l^{2} \sqrt{n}}$. Substituting $T(r)$ into the equation, we have

$$
\lambda(n) \leq \frac{r T(r)}{l^{2} \sqrt{n}}=\frac{P_{t} \min \left(1, r^{-\alpha}\right)}{N_{0}+\sum_{k \in \mathcal{T} \backslash\{i\}} P_{t} l\left(x_{k}, x_{j}\right)} \frac{r}{l^{2} \sqrt{n}}
$$

where $\mathcal{T}$ denotes the set of concurrent transmission nodes and $l\left(x_{k}, x_{r}\right)$ denotes the path loss gain.

It is easily verified that $\min \left(r, r^{1-\alpha}\right)=O(1)$. Next We show that $\left(N_{0}+\sum_{k \in \mathcal{T} \backslash\{i\}} P_{t} l\left(x_{k}, x_{r}\right)\right) l^{2}=\Omega(1)$. If $l=$ $\Omega(1)$, the result is straightforward. If $l=o(1)$, there would be $\Theta\left(\frac{1}{l^{2}}\right)$ concurrent transmission nodes inside unit area around the receiver and the path loss gain between these nodes and the intended receiver is $\Theta(1)$. Therefore, the result holds.

Since the per-node throughput without the secrecy constraint is $O\left(\frac{1}{\sqrt{n}}\right)$, the per-node secrecy capacity can also be bounded by $O\left(\frac{1}{\sqrt{n}}\right)$ which indicates the optimality of our scheme.

\section{Colluding EAVESDroppers}

In previous section, the maximum SINR received by an independent eavesdropper can be suppressed by artificial noise generation. And it has already been shown that the per-node throughput does not entail lost, regardless of how many eavesdroppers are present in the network. However, if eavesdroppers are equipped with multiple antennas or multiple eavesdroppers can collude to decode the messages, is it still possible to 
ensure secrecy transmission and what is the network secrecy performance? We will focus on these problems in this section.

\section{A. Eavesdroppers with Multiple Antennas}

We start from the special case where every eavesdropper is equipped with $A(n)$ antennas but eavesdroppers do not collaborate with each other.

To get an intuitive insight, we assume that the eavesdropper can employ maximum ratio combining to maximize the SINR which means that the correlation across the antennas is ignored.

Theorem 3: If eavesdroppers are equipped with $A(n)$ antennas, the per-node secrecy capacity $\lambda_{s}(n)$ is $\Omega\left(\frac{1}{\sqrt{n}} A(n)^{-\frac{2}{\alpha}}\right)$.

Proof: Since eavesdroppers are equipped with $A(n)$ antennas, following the same argument of Lemma 1, the maximum rate that eavesdroppers can get is bounded as $R_{e} \leq c_{3} A(n) \frac{P_{t}}{P_{r}}\left(1+d_{r t}\right)^{\alpha}$. Because eavesdroppers don't transmit any noise, the rate that legitimate receivers can get remains the same as the following

$$
R(d) \geq c_{2} P_{t}(c(d+1))^{-\alpha} .
$$

Because of $R_{s}(d)=R(d)-R_{e}$, it is obvious that there exists a constant $c_{3}^{\prime}$, such that when $P_{r}$ equals to $c_{3}^{\prime} A(n) d^{2 \alpha}$, $R_{s}(d)$ can be greater than $\frac{1}{2} R(d)$. Hence the secrecy rate is $\Omega\left(d^{-\alpha}\right)$. To hold equation (6), $k$ should be in the order of $\Theta\left(P_{r}{ }^{\frac{1}{\alpha}}\right)$ which is equal to $\left(A(n)^{\frac{1}{\alpha}} d^{2}\right)$. So the secrecy rate in each cell should be $\Omega\left(d^{-\alpha-4} A(n)^{-\frac{2}{\alpha}}\right)$. When the transmission is on the highway phase, the per-node secrecy capacity is $\Omega\left(\frac{1}{\sqrt{n}} A(n)^{-\frac{2}{\alpha}}\right)$. When the transmission is on the delivery phase, the per-node secrecy capacity is $\Omega\left(\log ^{-\alpha-5} n A(n)^{-\frac{2}{\alpha}}\right)$. Hence, the per-node secrecy capacity is $\Omega\left(\frac{1}{\sqrt{n}} A(n)^{-\frac{2}{\alpha}}\right)$. From this result, we can see that there is a tradeoff between the number of a eavesdropper' antennas and the capacity of legitimate nodes.

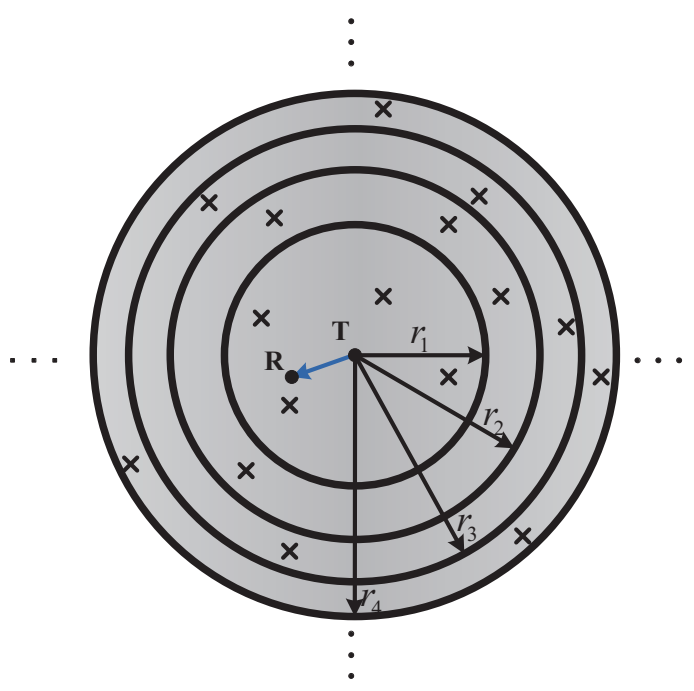

Fig. 2: An illustration of network partition to bound the upper bound of SINR received by eavesdroppers.

\section{B. Secrecy Capacity for Colluding Eavesdroppers}

To get a fundamental insight on how the colluding eavesdroppers will affect the secrecy transmission, we assume that all eavesdroppers in the network can collaborate to decode the messages and maximum ratio combining is adopted to maximize the SINR eavesdroppers obtained. Hence we can regard all eavesdroppers as a super-eavesdropper.

Assume that eavesdroppers are poisson distributed with parameter $\psi_{e}(n)$ in the network. For a given transmitterreceiver pair, we partition the network into disjoint rings with a same size of $f(n)$. The transmitter is at the center of all these rings. Let $r_{i}$ be the external diameter of the $i$ th ring. Since $f(n)=\pi r_{1}^{2}=\pi\left(r_{i}^{2}-r_{i-1}^{2}\right)$ for any $i>1$, we have $r_{i}=\sqrt{i} r_{1}$ for any $i \geq 1$. Denote $\Phi_{e i}$ as the set of eavesdroppers located inside the $i$-th ring. Hence the number of eavesdropper $N_{e i}$ in $\Phi_{e i}$ is a poisson variable with parameter $\psi_{e}(n) f(n)$. Recalling Lemma 4, we have

$$
P\left(\frac{1}{2} f(n) \psi_{e}(n) \leq N_{e i} \leq 2 f(n) \psi_{e}(n), \forall i\right)=1,
$$

when $f(n) \psi_{e}(n) \geq \log _{4 / e} n$ and $f(n)=\Omega(1)$.

Notice that the distance between the transmitter and eavesdroppers is at least $r_{i-1}$, the signal power received by eavesdroppers in the $i$-th ring is at most $P_{t} r_{i-1}^{-\alpha}$ for any $i \geq 2$. For each $\psi_{e}(n)$, we choose $f(n)$ such that $f(n) \psi_{e}(n) \geq \log _{4 / e} n$ and $f(n)=\Omega(1)$. Denote $\operatorname{SINR}_{e i}$ as the SINR received by eavesdroppers in the $i$-th ring. Taking the summation of the SINR received by the eavesdroppers in all the rings up, we have

$$
\begin{aligned}
& \operatorname{SINR}_{e} \leq \sum_{i} \operatorname{SINR}_{e i} \\
& =\sum_{j \in \Phi_{e 1}} \operatorname{SINR}_{1 j}+\sum_{i=2}^{+\infty} \sum_{j \in \Phi_{e i}} \operatorname{SINR}_{i j} \\
& \leq 2 f(n) \psi_{e}(n) \overline{\operatorname{SINR}_{e 1}}+\sum_{i=2}^{+\infty} 2 f(n) \psi_{e}(n) \overline{\operatorname{SINR}_{e i}} \\
& \leq 2 f(n) \psi_{e}(n) \frac{P_{t}}{P_{r}}\left(1+d_{r t}\right)^{\alpha}+\sum_{i=2}^{+\infty} 2 f(n) \psi_{e}(n) \frac{P_{t} r_{i-1}^{-\alpha}}{N_{0}} \\
& =2 \pi \psi_{e}(n)\left(r_{1}^{2} \frac{P_{t}}{P_{r}}\left(1+d_{r t}\right)^{\alpha}+\frac{P_{t}}{N_{0}} r_{1}^{2-\alpha} \sum_{i=1}^{+\infty} i^{-\frac{\alpha}{2}}\right)
\end{aligned}
$$

where the third row of this inequality follows from Lemma 1 and note that $\sum_{i=1}^{+\infty} i^{-\frac{\alpha}{2}}$ converges since $\alpha>2$.

Case 1: When the transmission is on the highway phase which means $d_{r t}=\Theta(1)$, substituted into Equation (8), it is obvious that there is a constant $c_{4}$ satisfying $R_{e} \leq$ $c_{4} \psi_{e}(n)\left(r_{1}^{2} / P_{r}+r_{1}^{2-\alpha}\right)$. As is shown in Lemma 2, the rate $R(d)$ received by the intended receiver can be $\Theta(1)$. Note that there are two constraints in the derivation of Equation (9), i.e., $f(n) \psi_{e}(n) \geq \log _{4 / e} n$ and $f(n)=\Omega(1)$. With $r_{1}=\max \left(\Omega(1), \Theta\left(\psi_{e}(n)^{\frac{1}{\alpha-2}}\right)\right)$ and $P_{r}=\Theta\left(\psi_{e}(n) r_{1}^{2}\right)$, the secure transmission can be guaranteed and secure rate each node in the highway can transmit is $\Omega\left(\frac{1}{k^{2}}\right)$ where $k=\Theta\left(P_{r}^{\frac{1}{\alpha}}\right)$ is the concurrent transmission range.

Hence if $\psi_{e}(n)=\Omega\left(\log \frac{\alpha-2}{\alpha} n\right), P_{r}=\psi_{e}(n) r_{1}^{2}=$ 
$\Theta\left(\psi_{e}(n)^{\frac{\alpha}{\alpha-2}}\right)$. The secure rate each node in the highway can transmit is $\Omega\left(\psi_{e}(n)^{-\frac{2}{\alpha-2}}\right)$. Since the traffic load at each node in the highway is at most $O(\sqrt{n})$, the per-node throughput should be $\Omega\left(\frac{1}{\sqrt{n}} \psi_{e}(n)^{-\frac{2}{\alpha-2}}\right)$. If $\psi_{e}(n)=O\left(\log \frac{\alpha-2}{\alpha} n\right)$, with similar argument, the noise generation power can be $\Theta(\log n)$ and we can obtain per-node secrecy capacity of $\Omega\left(\frac{1}{\sqrt{n}} \log ^{-\frac{2}{\alpha}} n\right)$.

Case 2: When the transmission is on the draining and delivery phases where $d_{r t}=\Theta(\log n)$, there exists a constant $c_{5}$ such that $\operatorname{SINR}_{e} \leq c_{5} \psi_{e}(n)\left(r_{1}^{2} \log ^{\alpha} n / P_{r}+r_{1}^{2-\alpha}\right)$. The rate that a legitimate receiver can obtained is $\log _{1}^{-\alpha} n$. Similar to case 1 , choosing $r_{1}=\max \left(\Omega(1), \Theta\left(\psi_{e}(n)^{\frac{1}{\alpha-2}} \log ^{\frac{\alpha}{\alpha-2}} n\right)\right)$ and $P_{r}=\Theta\left(\psi_{e}(n) r_{1}^{2} \log ^{2 \alpha} n\right)$, the secure transmission could be guaranteed and secure rate $R_{s}$ allocated at each cell is $\Omega\left(\frac{1}{k^{2} \log ^{\alpha} n}\right)$, where $k=\Theta\left(P_{r}^{\frac{1}{\alpha}}\right)$. When $\psi_{e}(n)=\Omega(1), P_{r}=\Theta\left(\psi_{e}(n)^{\frac{\alpha}{\alpha-2}} \log ^{\frac{2 \alpha^{2}}{\alpha-2}} n\right)$ and $R_{s}=$ $\Omega\left(\psi_{e}(n)^{-\frac{2}{\alpha-2}} \log ^{-\alpha} n \log ^{-\frac{4 \alpha}{\alpha-2}} n\right)$. Since there are at most $\log n$ legitimate nodes inside a cell, the per-node secrecy capacity is bounded by $\Omega\left(\psi_{e}(n)^{-\frac{2}{\alpha-2}} \log ^{-\alpha-1} n \log ^{-\frac{4 \alpha}{\alpha-2}} n\right)$. When $\psi_{e}(n)=O(1)$, using similar technique, we can obtain that the per-node secrecy capacity is at least a polylog(n) factor. Therefore, the bottleneck lies in the highway phase.

Combining these two cases, we present the following theorem which demonstrates the tradeoff between the secrecy capacity and the tolerable eavesdroppers' density.

Theorem 4: Consider the wireless network $\mathscr{B}$ where legitimate nodes and eavesdroppers are independent poisson distributed with parameter 1 and $\psi_{e}(n)$ respectively, the pernode secrecy capacity is

$$
\lambda_{s}(n)=\left\{\begin{array}{ll}
\Omega\left(\frac{1}{\sqrt{n}} \psi_{e}(n)^{-\frac{2}{\alpha-2}}\right), & \psi_{e}(n)=\Omega\left(\log \frac{\alpha-2}{\alpha} n\right) \\
\Omega\left(\frac{1}{\sqrt{n}} \log ^{-\frac{2}{\alpha}} n\right), & \psi_{e}(n)=O\left(\log ^{\frac{\alpha-2}{\alpha}} n\right)
\end{array} .\right.
$$

Intuitively, when $\psi_{e}(n)=o\left(n^{-1}\right)$, the number of eavesdroppers will be at most 1 w.h.p. according to the weak law of large numbers. Hence, the secrecy capacity will be $\Omega\left(\frac{1}{\sqrt{n}}\right)$ with Theorem 2 which is much higher than the results in Theorem 3. The main reason is that the inequality $f(n) \psi_{e}(n) \geq \log _{4 / e} n$ should be satisfied throughout the proof of Theorem 3. Therefore, the noise generation power should be $\Theta(\log n)$ which will degrade the throughput performance. We re-investigate this problem from another perspective in the following context.

Lemma 6: When the intensity of the eavesdroppers is $\psi_{e}(n)=O\left(n^{-\beta}\right)$ for any constant $\beta>0$, partitioning the network into disjoint regions with constant size $h$ and denoting by $N_{e i}$ the number of nodes inside region $i$, we have

$$
P\left(N_{e i} \leq v, \forall i\right)=1,
$$

where $v=\left\lceil\frac{1}{\beta}\right\rceil+1$.

Proof: Let $N_{e}$ be the maximum number of $N_{e i}$ and $\psi$ be the expected number of $N_{e i}$. Hence $\psi=h \psi_{e}(n)$ and we can further get

$$
\begin{aligned}
P\left(N_{e i} \geq v\right) & =\sum_{i=v}^{\infty} \frac{\psi^{i} e^{-\psi}}{i !} \\
& \leq \frac{\psi^{v} e^{-\psi}}{v !}\left(1+\psi+\psi^{2}+\psi^{3}+\ldots\right) \\
& \leq \frac{\psi^{k} e^{-\psi}}{v !} \frac{1}{1-\psi /(v+1)} \rightarrow 0 .
\end{aligned}
$$

Using the union bound, we have

$$
\begin{aligned}
P\left(N_{e} \geq v\right) & \leq \frac{n}{h} \frac{\psi^{v} e^{-\psi}}{v !} \frac{1}{1-\psi /(v+1)} \\
& \leq \frac{n^{1-v \beta}}{h} \frac{h^{v} e^{-\psi}}{v !} \frac{1}{1-\psi /(v+1)} \rightarrow 0
\end{aligned}
$$

as $n$ goes to infinity.

Theorem 5: If eavesdroppers are poisson-distributed in the network with intensity $\psi_{e}(n)=O\left(n^{-\beta}\right)$ for any constant $\beta>$ 0 , the per-node secrecy capacity is $\Omega\left(\frac{1}{\sqrt{n}}\right)$.

Proof: To compute the SINR of the eavesdropper system, we divide the network into two parts. One is the circle which is at most $r_{1}$ distance from the transmitter, the other is the rest of the network. There are at most $v \pi r_{1}^{2}$ eavesdroppers in the circle where $v$ is a constant as is shown in Lemma 5 and the SINR received by each eavesdropper is upper bounded by the results in Lemma 1. Thus, the cumulative SINR received by eavesdroppers can be calculated as

$$
\begin{aligned}
\operatorname{SINR}_{e} & \leq v \pi r_{1}^{2} \frac{P_{t}}{P_{r}}\left(1+d_{r t}\right)^{\alpha}+\int_{r_{1}}^{\infty} \frac{P_{t} r^{-\alpha}}{N_{0}} 2 \pi r v d r \\
& =v \pi r_{1}^{2} \frac{P_{t}}{P_{r}}\left(1+d_{r t}\right)^{\alpha}+\frac{P_{t} 2 \pi v r_{1}^{2-\alpha}}{N_{0}(\alpha-2)} .
\end{aligned}
$$

When packets are delivered along the highway, where the distance between T-R pairs is $d_{r t}=\Theta(1)$ and the rate $R(d)$ is a positive constant, there exists constants $c_{6}$ and $c_{7}$ such that $R_{e} \leq \operatorname{SINR}_{e} \leq \frac{1}{2} R(d)$ when $r_{1}=c_{6}$ and $P_{r}=c_{7} r_{1}^{2}$. As shown in Equation (6), the concurrent transmission range $k$ is $\Theta\left(P_{r}^{\frac{1}{\alpha}}\right)$ and hence is $\Theta(1)$. So the secrecy rate each node on the highway can transmit is $\Theta(1)$. Since the node should relay at most $\Theta(\sqrt{n})$ nodes' traffic, the per-node secrecy capacity is $\Omega\left(\frac{1}{\sqrt{n}}\right)$.

When the transmission is on the draining and delivery cases where $d_{r t}=\Theta(\log n)$, the rate each cell can transmit is $\Theta\left(\log ^{-\alpha} n\right)$ as shown in Lemma 2. By choosing $r_{1}=c_{8} \log ^{\frac{\alpha}{\alpha-2}} n$ and $P_{r}=c_{9} r_{1}^{2} \log ^{2 \alpha} n$ where $c_{8}$ and $c_{9}$ are both constants, we can obtain $\operatorname{SINR}_{e} \leq$ $\frac{1}{2} R(d)$. The concurrent transmission range $k$ is $\Theta\left(P_{r}^{\frac{1}{\alpha}}\right)=$ $\Theta\left(\log \frac{2 \alpha-2}{\alpha-2} n\right)$. Hence the secrecy throughput each cell can transmit is $\Theta\left(\log ^{-\frac{4 \alpha-4}{\alpha-2}} n \log ^{-\alpha} n\right)$. Since there are at most $\Theta(\log n)$ nodes in the cell, the per-node secrecy capacity is $\Omega\left(\log ^{-\frac{4 \alpha-4}{\alpha-2}} n \log ^{-\alpha-1} n\right)$.

Combining the results above, we conclude this theorem.

With Theorem 4 and Theorem 5, the per-node secrecy 


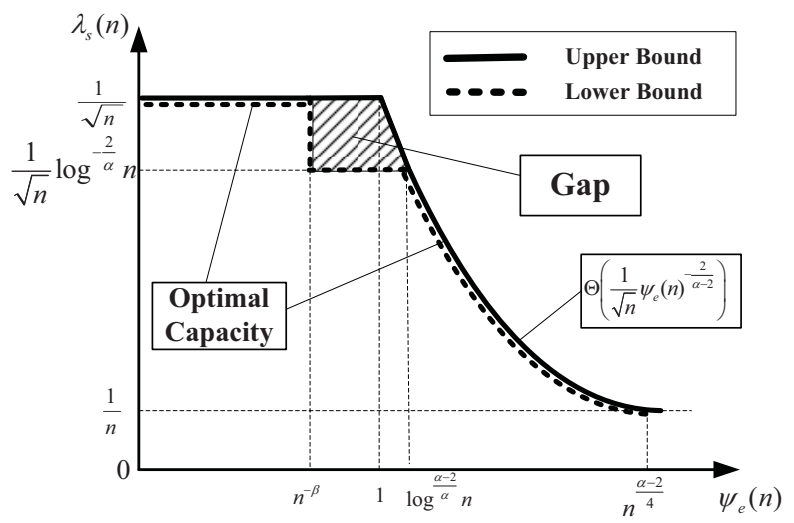

Fig. 3: An illustration of both upper bound and lower bound of secrecy capacity in large-scale networks. The scales of the axes are in terms of the orders in $n$.

capacity can be summarized as follows

$\lambda_{s}(n)= \begin{cases}\Omega\left(\frac{1}{\sqrt{n}} \psi_{e}(n)^{-\frac{2}{\alpha-2}}\right) & \psi_{e}(n)=\Omega\left(\log \frac{\alpha-2}{\alpha} n\right) \\ \Omega\left(\frac{1}{\sqrt{n}} \log ^{-\frac{2}{\alpha}} n\right) & \psi_{e}(n)=\left[\Omega\left(n^{-\beta}\right), O\left(\log \frac{\alpha-2}{\alpha} n\right)\right] \\ \Omega\left(\frac{1}{\sqrt{n}}\right) & \psi_{e}(n)=O\left(n^{-\beta}\right)\end{cases}$

for any constant $\beta>0$.

\section{The Optimality of Our Scheme}

In previous subsection, we have derived the lower bounds of the network secrecy capacity in collaborating case. However, the upper bound of the network secrecy capacity still remains unknown. We will focus on the upper bound in this subsection.

It is also assumed that legitimate nodes do not cooperate to generate artificial noise here while the cooperative mode will be discussed in the next subsection.

Theorem 6: Consider the wireless network $\mathscr{B}$ where legitimate nodes and eavesdroppers are independent poisson distributed with parameter 1 and $\psi_{e}(n)$ respectively, the pernode secrecy capacity is

$$
\lambda_{s}(n)= \begin{cases}O\left(\frac{1}{\sqrt{n}} \psi_{e}(n)^{-\frac{2}{\alpha-2}}\right) & \psi_{e}(n)=\Omega(1) \\ O\left(\frac{1}{\sqrt{n}}\right) & \psi_{e}(n)=O(1)\end{cases}
$$

Proof: When the transmission is on the highway, we assume that the concurrent transmission range is $k$ and partition the network into disjoint subsquares with size $k \times k$. Denote the two squares with length $\frac{3 k}{4}$ and length $\frac{k}{4}$ whose centers are both at node $i$ as $A_{1 i}$ and $A_{2 i}$ respectively. Let the region $A_{1 i}-A_{2 i}$ be $A_{i}$. Denote the number of eavesdroppers located in $A_{i}$ as $N_{e i}$ where $i$ ranges from 1 to $\frac{n}{k^{2}}$. Since the expectation of the number of eavesdroppers located in all the regions $A_{i}$ is $\frac{n}{2} \psi_{e}(n)$, there are at least $\frac{n}{4} \psi_{e}(n)$ eavesdroppers in all the regions $A_{i}$ when $\psi_{e}(n) \geq \frac{\log _{4 / e} n}{n}$ according to Lemma 4 . Hence there exists a $i$ such that $N_{e i}$ will be greater than $\frac{k^{2}}{4} \psi_{e}(n)$.

Consider a specific eavesdropper $j$ in region $A_{i}$. Since the minimum distance between eavesdropper $j$ and the eight closest concurrent transmission is at least $\frac{k}{4}$ and the next

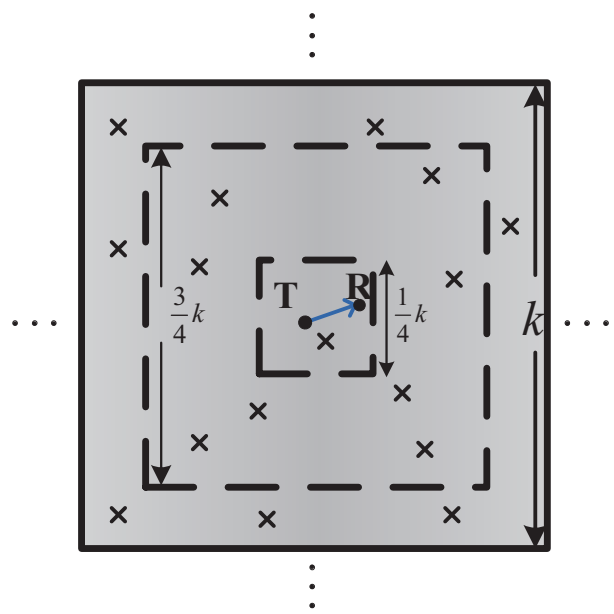

Fig. 4: An illustration of network partition to bound the lower bound of SINR received by eavesdroppers.

sixteen is at least $\frac{5 k}{4}$, the interference eavesdropper $j$ suffers from can be bounded as follows.

$$
\begin{aligned}
I_{j} & \leq \sum_{c=1}^{\infty} 8 c\left(P_{t}+P_{r}\right)\left(\frac{k}{4}+(c-1) k\right)^{-\alpha} \\
& =\left(P_{t}+P_{r}\right)(k)^{-\alpha} \sum_{c=1}^{\infty} 8 c\left(c-\frac{3}{4}\right)^{-\alpha} \\
& \leq c_{10} P_{r} k^{-\alpha}
\end{aligned}
$$

where $c_{10}$ is a constant.

As is shown in Theorem $1, k$ should be $\Omega\left(P_{r}^{\frac{1}{\alpha}}\right)$. Therefore, the interference eavesdropper $j$ suffers from can be bounded by a constant. The maximum distance between eavesdropper $j$ and the closest transmitter is at most $\frac{3 k}{4}$. Hence, the SINR received by all the eavesdroppers in region $A_{i}$ can be lower bounded by

$$
\begin{aligned}
\operatorname{SINR}_{e} & \geq \sum_{j} \frac{S_{j}}{N_{0}+I_{j}} \\
& \geq N_{e i} \frac{\left(\frac{3 k}{4}\right)^{-\alpha}}{N_{0}+\overline{I_{j}}} \\
& \geq c_{11} \psi_{e}(n) k^{2-\alpha},
\end{aligned}
$$

when $c_{11}$ is a constant.

Since the rate at which each T-R pair can transmit is $\Theta(1)$, we should choose $k=\Omega\left(\psi_{e}(n)^{\frac{1}{\alpha-2}}\right)$ to ensure the secrecy of transmission. Note that there are $k^{2}$ cells in each subsquare taking turn to transmit and each node in the highway should carry the traffic load of $\Theta(\sqrt{n})$ nodes. Hence the per-node secrecy capacity is at most $O\left(\frac{1}{k^{2} \sqrt{n}}\right)=O\left(\frac{1}{\sqrt{n}} \psi_{e}(n)^{-\frac{2}{\alpha-2}}\right)$.

Note that according to Theorem 3, the per-node secrecy capacity is at most $O\left(\frac{1}{\sqrt{n}}\right)$. Therefore, the upper bound of secrecy capacity is $\min \left(O\left(\frac{1}{\sqrt{n}}\right), O\left(\frac{1}{\sqrt{n}} \psi_{e}(n)^{-\frac{2}{\alpha-2}}\right)\right)$.

From the above analysis, we can obtain some intuitive insight on the main constraint of secrecy capacity. While the legitimate receivers can generate artificial noise to affect the near-by eavesdroppers' channels, eavesdroppers which lie in the middle of two concurrent transmission T-R pairs may not 
be affected in order sense by the generated noise. Therefore, there is a clear tradeoff between the density of eavesdroppers and the concurrent transmission opportunities.

Compared with Theorem 4, our scheme achieves optimal secrecy capacity when $\psi_{e}(n)=\Omega\left(\log \frac{\alpha-2}{\alpha} n\right)$ and $\psi_{e}(n)=$ $O\left(n^{-\beta}\right)$ for any constant $\beta>0$. And when $\psi_{e}(n)=$ $O\left(\log \frac{\alpha-2}{\alpha} n\right)$, our scheme is close to the optimal one. A more clear picture of such optimality is illustrated in Fig. 3. And we leave it as our future work to further close this gap.

\section{Discussion on the Upper Bound}

Since the overhead to coordinate the generation of artificial noise between legitimate nodes maybe too large, we don't consider the scenario when legitimate nodes cooperate to generate artificial noise to enhance the secret transmission throughout this paper. The scheme proposed in previous Part $\mathrm{B}$ and Part $\mathrm{C}$ has also shown its great potential to improve secrecy capacity. However, it is still of great interest to see what is the secrecy capacity when legitimate nodes can cooperate and how to achieve it. Therefore, we consider the cooperative mode here and only present the key analysis in this part to avoid redundancy with previous sections.

Theorem 7: Consider the wireless network $\mathscr{B}$ where legitimate nodes and eavesdroppers are independent poisson distributed with parameter 1 and $\psi_{e}(n)$ respectively, the pernode secrecy capacity is

$$
\lambda_{s}(n)= \begin{cases}O\left(\frac{1}{\sqrt{n}} \psi_{e}(n)^{-\frac{2}{\alpha_{1}}}\right) & \psi_{e}(n)=\Omega(1) \\ O\left(\frac{1}{\sqrt{n}}\right) & \psi_{e}(n)=O(1)\end{cases}
$$

when legitimate nodes can cooperate to generate artificial noise and $\alpha_{1}=\alpha-\frac{\alpha}{\alpha-1}$.

Proof: Artificial noise is helpful only when the eavesdroppers' SINR is decreased more than the legitimate receivers'. Assume that a legitimate node which is $d=\Omega(1)$ distance away from legitimate receivers can generate artificial noise with power $P_{d}$. Since there are $\Theta\left(d^{2}\right)$ legitimate nodes which are $\Theta(d)$ distance away from legitimate receivers, the noise that the legitimate receiver will suffer is $\Theta\left(d^{2} \cdot P_{d} \cdot d^{-\alpha}\right)$. Therefore, when $P_{d}$ is larger than $\Theta\left(d^{\alpha-2}\right)$, the SINR legitimate receiver obtained will suffer a same loss with eavesdroppers'.

Divide the network into rings according to the distance $\left(d_{i}=\right.$ $2^{i}$ ) to the legitimate receiver. Therefore, in the i-th ring, there are about $\Theta\left(\psi_{e}(n) d_{i}^{2}\right)$ eavesdroppers. The noise each eavesdropper suffer is at most $\Theta\left(d_{i}^{\alpha-2}+P_{r} d_{i}^{-\alpha}\right)$ where $P_{r}$ is at most $\Theta\left(k^{\alpha}\right)$ similar to previous subsection. Hence, the SINR received by all eavesdroppers can be lower bounded by

$$
\begin{aligned}
\operatorname{SINR}_{e} & \geq c_{12} \sum_{d_{i}} \frac{\psi_{e}(n) d_{i}^{2} d_{i}^{-\alpha}}{k^{\alpha} d_{i}^{-\alpha}+d_{i}^{\alpha-2}} \\
& \geq \frac{c_{12}}{2} \sum_{d_{i} \leq k_{1}} \frac{\psi_{e}(n) d_{i}^{2-\alpha}}{k^{\alpha} d_{i}^{-\alpha}}+\frac{c_{12}}{2} \sum_{d_{i}>k_{1}} \frac{\psi_{e}(n) d_{i}^{2-\alpha}}{d_{i}^{\alpha-2}} \\
& \geq \frac{c_{12}}{2} \psi_{e}(n) k^{\frac{\alpha}{\alpha-1}-\alpha},
\end{aligned}
$$

when $c_{12}$ is a constant and $k_{1}=k^{\frac{\alpha}{2 \alpha-2}}$. Following similar argument in Part $\mathrm{C}$, we conclude this theorem.
According to the main idea of the upper bound, we can design a corresponding scheme to achieve the upper bound except a polylog(n) factor.

Since the network is divided into rings with $d_{i}=2^{i}$, there are at most $\Theta(\log n)$ rings in the network. Therefore, the cumulative noise suffered by the intended receiver is at most $\Theta(\log n)$. To ensure the rate that legitimate receiver can obtain is larger than that of eavesdroppers', there are two possible solutions. The artificial noise generated by the cooperative nodes can be scaled down by a $\Theta(\log n)$ factor. On the other hand, we may also increase the $P_{r}$ to further decrease the eavesdroppers' signals. After delicate computation, it can be shown that the first solution has a better performance and therefore we only present the proof of the first solution in the following. Since we adopt the first solution, $P_{d}=d^{\alpha-2} \log ^{-1} n$ and the rate that the legitimate T-R pair can transmit remains as a constant. Divide the network into $k^{2}$ disjoint subsquares. The cumulative SINR that eavesdroppers can obtain inside the intended legitimate receiver's subsquare can be bounded by

$$
\begin{aligned}
\operatorname{SINR}_{e 1} & \leq c_{13} \sum_{d_{i}} \frac{\psi_{e}(n) d_{i}^{2-\alpha}}{k^{\alpha} d_{i}^{-\alpha}+d_{i}^{\alpha-2} \log ^{-1} n \log ^{-\alpha} n} \\
& \leq c_{13} \sum_{d_{i} \leq k_{2}} \frac{\psi_{e}(n) d_{i}^{2-\alpha}}{k^{\alpha} d_{i}^{-\alpha}}+c_{13} \sum_{d_{i}>k_{2}} \frac{\psi_{e}(n) d_{i}^{2-\alpha}}{d_{i}^{\alpha-2} \log ^{-\alpha-1} n} \\
& \leq 4 c_{13} \psi_{e}(n) \log ^{\frac{\alpha+1}{\alpha-1}} n \cdot k^{\frac{\alpha}{\alpha-1}-\alpha},
\end{aligned}
$$

when $c_{13}$ is a constant and $k_{2}=k^{\frac{\alpha}{2 \alpha-2}}(\log n)^{\frac{\alpha+1}{2 \alpha-2}}$. Note that the main difference between Equation (19) and (20) is a $\log ^{\alpha} n$ parameter. This is a path loss gain because the maximum distance between an eavesdropper and a legitimate node generating noise is $\log n$. It can also be shown that the cumulative SINR that eavesdroppers can obtain inside other subsquares is smaller than $\operatorname{SINR}_{e 1}$. Therefore, using similar approach in Part B, the secrecy capacity is

$$
\lambda_{s}(n)=\Omega\left(\frac{1}{\sqrt{n}} \psi_{e}(n)^{-\frac{2}{\alpha_{1}}} \log ^{\frac{-2(\alpha+1)}{\alpha_{1}(\alpha-1)}} n\right)
$$

when $\psi_{e}(n) k_{2}^{2}=\Omega(\log n)$.

\section{Secrecy Capacity in Dense Networks AND RANDOM NETWORKS}

\section{A. Secrecy Capacity in Dense Networks}

In previous section, we have considered secrecy transmissions in extended networks. Now we will extend it to dense networks where nodes are poisson distributed in a unit square. On surface, the difference seems to be only a scale factor of $\sqrt{n}$ and all the results can be applied to the dense networks directly. However, we note that this is not the case because the path loss gain in extended networks is bounded while it is unbounded in dense networks. Consider that an eavesdropper is quite close to the transmitter, the SINR at the eavesdropper will be quite large. Hence we should first consider the minimum distance between eavesdroppers and the transmitters which is denoted by $b$. Let $N_{e}$ be the number of eavesdroppers in the dense network. When $n \pi b^{2} N_{e}=o(1)$, 
the region inside the circles centered at transmitters with diameter $b$ will be empty of eavesdroppers.

Conducting similar derivation in Theorem 1 , we can obtain that $\operatorname{SINR}_{e} \leq c_{1} \frac{P_{t}}{P_{r}}\left(\frac{1}{b \sqrt{n}}\right)^{\alpha}$ and $\operatorname{SINR}_{l} \geq c_{2} P_{t}(\sqrt{n})^{\alpha}$ when the concurrent transmission range $k$ is greater than $P_{r}^{\frac{1}{\alpha}}$. To assure the transmission is secret, we choose $P_{r}=\Theta\left(\left(\frac{1}{b n}\right)^{\alpha}\right)$. Hence, the concurrent transmission range is $\Theta\left(\frac{1}{b n}\right)$ and the per-node secrecy capacity is $\Omega\left(\frac{1}{\sqrt{n}}\left(\frac{b n}{\sqrt{n}}\right)^{2}\right)=\Omega\left(\frac{1}{\sqrt{n} N_{e}}\right)$.

Compared with the results in extended networks, the secrecy concern has a much larger impact on dense networks. Therefore, it is a tempting future work to study how to improve secrecy capacity in dense networks, e.g., mobility may help.

\section{B. Secrecy Capacity in Random Networks}

The difference between Poisson distributed network and the random network lies in that with network divided into multiple regions, the numbers of nodes inside a specific region in the former one are mutually independent poisson variables whereas it is not the case in the latter one. Hence it is often computable in poisson networks due to this independence property. In contrast, this does not necessarily hold in random networks since the total number of nodes in the network is given. Thus, the number of nodes inside a given region may affect the distribution probability of that in other regions. However, it is shown in [29] that random networks will converge to Poisson scenarios as $n$ goes to infinity which is also proved in the following from another perspective. We note that while the following result is straight forward at the first glance although it is not, the lemma given below has its own value and the proof here is different from that in [29]. Hence, our results still hold when applied to random networks.

Lemma 7: Partition the network into disjoint cells with equal size 1 , if there are $k$ nodes randomly distributed in the network, then the probability each cell has $i$ nodes is

$$
C_{k}^{i}\left(\frac{1}{n}\right)^{i}\left(1-\frac{1}{n}\right)^{k-i}
$$

Proof: Denote the number of cells that has $i$ nodes in it as $T_{k}^{\prime}(i)$ and the expectation of $T_{k}^{\prime}(i)$ as $T_{k}(i)$. Consider there are $k$ nodes in the network and a new node joins in. If the new node joins in a cell with $i$ nodes, $T_{k+1}^{\prime}(i)$ should be $T_{k}^{\prime}(i)-1$ and $T_{k+1}^{\prime}(i+1)$ should be $T_{k}^{\prime}(i+1)+1$. Hence, we have

$$
\begin{gathered}
T_{k+1}^{\prime}(0)= \begin{cases}T_{k}^{\prime}(0) & \frac{n-T_{k}^{\prime}(0)}{n} \\
T_{k}^{\prime}(0)-1 & \frac{T_{k}^{\prime}(0)}{n}\end{cases} \\
T_{k+1}^{\prime}(i)= \begin{cases}T_{k}^{\prime}(i)-1 & \frac{T_{k}^{\prime}(i)}{n} \\
T_{k}^{\prime}(i) & \frac{n-T_{k}^{\prime}(i)-T_{k}^{\prime}(i-1)}{n} \\
T_{k}^{\prime}(i)+1 & \frac{T_{k}^{\prime}(i-1)}{n}\end{cases}
\end{gathered}
$$

for all $i \geq 2$ and $i \leq k$, where the latter part of the equations is the probability of given event.

$$
T_{k+1}^{\prime}(k+1)=\left\{\begin{array}{ll}
0 & \frac{n-T_{k}^{\prime}(k)}{n} \\
1 & \frac{T_{k}^{\prime}(k)}{n}
\end{array} .\right.
$$

Taking expectation of both sides, we have

$$
\left\{\begin{array}{l}
T_{k+1}(0)=T_{k}(0)\left(1-\frac{1}{n}\right) \\
T_{k+1}(i)=\left(1-\frac{1}{n}\right) T_{k}(i)+\frac{1}{n} T_{k}(i-1) \\
T_{k+1}(k+1)=\frac{1}{n} T_{k}(k)
\end{array} .\right.
$$

Note that $T_{0}(0)=n, T_{1}(0)=n-1$ and $T_{1}(1)=1$, using mathematical induction, we have

$$
T_{k}(i)=n C_{k}^{i}\left(\frac{1}{n}\right)^{i}\left(1-\frac{1}{n}\right)^{k-i} .
$$

Since $n$ cells are the same, we conclude this lemma. When $n$ and $k$ both goes to infinity, the probability converges to a poisson variable with parameter $\frac{k}{n}$.

\section{Secrecy Capacity under Active Attacks}

We now consider the static ad hoc networks under jamming attacks in which jammers keep sending out radio waves to interrupt the legitimate nodes. This will cause additional interference and hence may degrade the performance of legitimate nodes. For simplicity, we assume the power utilized by all the jammers is the same, denoted by $P_{m}$ and $P_{m}=\Theta(1)$.

Assume that jammers are poisson distributed with parameter $\psi_{m}(n)$ in the network and the set of jammers is denoted by $\Phi_{m}$. For a given transmitter-receiver pair, we partition the network into disjoint rings with a same size of $f(n)$. The receiver is at the center of all these rings. Let $r_{i}$ be the external diameter of the $i$ th ring. Since $f(n)=\pi r_{1}^{2}=\pi\left(r_{i}^{2}-r_{i-1}^{2}\right)$ for any $i>1$, we have $r_{i}=\sqrt{i} r_{1}$ for any $i \geq 1$. Denote $\Phi_{m i}$ as the sets of eavesdroppers located inside $i$ th ring. Hence the number of eavesdropper $N_{m i}$ in $\Phi_{m i}$ is a poisson variable with parameter $\psi_{m}(n) f(n)$. Recalling Lemma 4, we have

$$
P\left(\frac{1}{2} f(n) \psi_{m}(n) \leq N_{m} \leq 2 f(n) \psi_{m}(n), \forall i\right)=1,
$$

when $f(n) \psi_{m}(n) \geq \log _{4 / e} n$ and $f(n)=\Omega(1)$.

Notice that the distance between the receiver and jammers is at least $r_{i-1}$, the interference at the receiver caused by jammers in the $i$-th ring is at most $P_{m} r_{i-1}^{-\alpha}$ for any $i \geq 2$. For each $\psi_{m}(n)$, we choose $f(n)$ such that $f(n) \psi_{m}(n) \geq \log _{4 / e} n$ and $f(n)=\Omega(1)$. Denote $\mathrm{I}_{m i}$ as the interference caused by jammers in the $i$-th ring. Taking the summation of interference caused by jammers in all the rings up, we have

$$
\begin{aligned}
\mathrm{I}_{m} & \leq \sum_{i} \mathrm{I}_{m i} \\
& =\sum_{j \in \Phi_{m 1}} \mathrm{I}_{1 j}+\sum_{i=2}^{+\infty} \sum_{j \in \Phi_{m i}} \mathrm{I}_{i j} \\
& \leq 2 f(n) \psi_{m}(n) \overline{\mathrm{I}_{m 1}}+\sum_{i=2}^{+\infty} 2 f(n) \psi_{m}(n) \overline{\mathrm{I}_{m i}} \\
& \leq 2 f(n) \psi_{m}(n) P_{m}+\sum_{i=2}^{+\infty} 2 f(n) \psi_{m}(n) P_{m} r_{i-1}^{-\alpha} \\
& =2 P_{m} f(n) \psi_{m}(n)\left(1+\sum_{i=1}^{+\infty} r_{i}^{-\alpha}\right) \\
& \leq c_{14} P_{m} f(n) \psi_{m}(n),
\end{aligned}
$$

where $c_{14}$ is a constant. 
Similar to Section III, the highway system is adopted to transmit the messages across the network. Different from Section III, the scheduling scheme will not vary with the density of jammers. Choose the concurrent transmission range $k$ as $2 d+1$ where $d$ is the distance between legitimate T-R pairs. According to Lemma 2, the interference $I(d)$ caused by other concurrent transmissions can be bounded as a constant. Hence, the rate that a receiver can obtain is

$$
\begin{aligned}
R(d) & =\Theta\left(\frac{P_{t} d^{-\alpha}}{N_{0}+I(d)+I_{m}}\right) \\
& \geq c_{15} \frac{P_{t} d^{-\alpha}}{f(n) \psi_{m}(n)},
\end{aligned}
$$

where $c_{15}$ is a constant.

Case 1: When $\psi_{m}(n) \leq \log n$, choosing $f(n)$ to satisfy the conditions in Lemma 4 , it is obvious to see that $R(d)=\Omega\left(\frac{d^{-\alpha}}{\log n}\right)$. When the transmission is on the highway phases which means $d=\Theta(1), R(d)=\Omega\left(\frac{1}{\log n}\right)$. Since the node on the highway should carry the load of at most $\sqrt{n}$ nodes according to Lemma 4, the per-node capacity is $\Omega\left(\frac{1}{\sqrt{n} \log n}\right)$. When the transmission is on the draining and delivery phases where $d=\Theta(\log n), R(d)=\Omega\left(\log ^{-\alpha-1} n\right)$. Since the concurrent transmission range is $\Theta(\log n)$ and this rate should be shared by at most $\log n$ nodes according to Lemma 3, the per-node capacity is $\Omega\left(\log ^{-\alpha-4} n\right)$. Combing the results above, the per-node capacity is $\Omega\left(\frac{1}{\sqrt{n} \log n}\right)$.

Case 2: When $\psi_{m}(n) \geq \log n$, choosing $f(n)=\Theta(1)$, we can see that $R(d)=\Omega\left(\frac{\bar{d}^{-\alpha}}{\psi_{m}(n)}\right)$ from Equation (28). Similar to the derivation in case 1 , the per-node capacity can be obtained which is $\Omega\left(\frac{1}{\sqrt{n} \psi_{m}(n)}\right)$ on the highway phase while it is $\Omega\left(\frac{\log ^{-\alpha-3} n}{\psi_{m}(n)}\right)$ on the draining and delivery phase. Hence, the per-node throughput is $\Omega\left(\frac{1}{\sqrt{n} \psi_{m}(n)}\right)$ when $\psi_{m}(n) \geq \log n$.

Combining these two cases, we present the following theorem which demonstrates the achievable capacity jamming attacks.

Theorem 8: Consider the wireless network $\mathscr{B}$ where legitimate nodes and jammers are independent poisson distributed with parameter 1 and $\psi_{m}(n)$ respectively, the per-node capacity is

$$
\lambda_{m}(n)=\left\{\begin{array}{ll}
\Omega\left(\frac{1}{\sqrt{n} \psi_{m}(n)}\right), & \psi_{m}(n) \geq \log n \\
\Omega\left(\frac{1}{\sqrt{n} \log n}\right), & \psi_{m}(n)<\log n
\end{array} .\right.
$$

According to Lemma 5, when $\psi_{m}(n)=o\left(n^{-\beta}\right)$ for any constant $\beta \geq 0$, the number of jammers will be upper bounded by $\Theta(1)$ w.h.p.. However, in previous lemma, the upper bound of the number of jammers is still $\Omega(\log n)$ and hence the result is not tight. Therefore, we re-investigate this problem in the following context.

Theorem 9: If jammers are poisson-distributed in the network with intensity $\psi_{m}(n)=O\left(n^{-\beta}\right)$ for any constant $\beta>0$, the per-node capacity is $\Omega\left(\frac{1}{\sqrt{n}}\right)$.

Proof: To compute the interference at the receiver, we divide the network into two parts. One is the circle which is at most $r_{1}$ distance from the receiver, the other is the rest of the network. There are at most $v \pi r_{1}^{2}$ jammers in the circle where $v=\left\lceil\frac{1}{\beta}\right\rceil+1$ is a constant as is shown in Lemma 5. Thus, the cumulative interference at the receiver can be calculated as

$$
\begin{aligned}
I_{m} & \leq v \pi r_{1}^{2} P_{m}+\int_{r_{1}}^{\infty} P_{m} r^{-\alpha} 2 \pi r v d r \\
& =v \pi r_{1}^{2} P_{m}+\frac{P_{m} 2 \pi v r_{1}^{2-\alpha}}{\alpha-2} .
\end{aligned}
$$

Hence, choosing $r_{1}=\Theta(1), I_{m}$ can be bounded as a constant. According to Equation (28), $R(d)=\Theta\left(\frac{P_{t} d^{-\alpha}}{N_{0}+I(d)+I_{m}}\right)=$ $\Omega\left(d^{-\alpha}\right)$. After similar derivation in the proof of Theorem 8 , we conclude this theorem.

Now, we can summarize the per-node capacity as follows.

$$
\lambda_{m}(n)= \begin{cases}\Omega\left(\frac{1}{\sqrt{n} \psi_{m}(n)}\right), & \psi_{m}(n)=\Omega(\log n) \\ \Omega\left(\frac{1}{\sqrt{n} \log n}\right), & \psi_{m}(n)=\left[\Omega\left(n^{-\beta}\right), O(\log n)\right] \\ \Omega\left(\frac{1}{\sqrt{n}}\right) & \psi_{m}(n)=O\left(n^{-\beta}\right)\end{cases}
$$

for any constant $\beta>0$.

Similar to the proof in Part C, Section IV, we can also prove the upper bound of capacity under the presence of jammers.

Theorem 10: Consider the wireless network $\mathscr{B}$ where legitimate nodes and jammers are independent poisson distributed with parameter 1 and $\psi_{m}(n)$ respectively, the per-node capacity is

$$
\lambda_{m}(n)=\left\{\begin{array}{ll}
O\left(\frac{1}{\sqrt{n}} \psi_{m}(n)\right. & \psi_{m}(n)=\Omega(1) \\
O\left(\frac{1}{\sqrt{n}}\right) & \psi_{m}(n)=O(1)
\end{array} .\right.
$$

\section{DISCUSSION}

\section{A. Details on the Self-interference Cancelation}

From the results above, we can see that the secrecy capacity is greatly improved by the adoption of self-interference cancelation. And recall that to use this technology, the distances between the receive antenna of legitimate node and the two noise generating antennas should differ half the wavelength. However, if the eavesdroppers can also use self-interference cancelation and the distances between their antennas and the two noise generating antennas differ one and half the wavelength or two and half the wavelength, the eavesdroppers would also suffer no artificial noise. To deal with this problem, we propose two methods in the following.

The first method that can be used is quite intuitive and effective. Just let the legitimate node revolve on its own axis, then only the position of the reception antenna of its own will suffer no artificial noise. And all the results in this paper still hold.

The other method maybe a little more complex and needs four noise generating antennas in each legitimate node. As shown in Fig. 5, $A_{1}, A_{2}$ and $B_{1}, B_{2}$ are the noise generating antennas and the reception antenna is located at the origin. The distances between $A_{1}$ and $A_{2}$ to the origin differ half the wavelength. So do $B_{1}$ and $B_{2}$. The noises generated by $A$ and $B$ are different. Hence, the positions that will suffer no artificial noise are contained in the shaded area as shown in Fig. 5. Also notice that the shaded area is located inside the legitimate node and hence all eavesdroppers will suffer the artificial noise. 


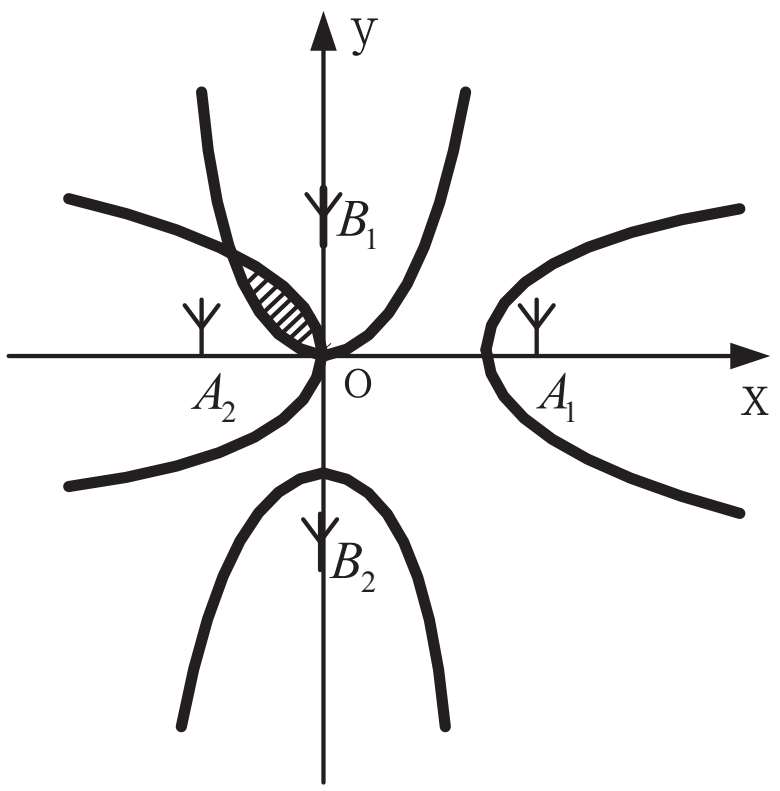

Fig. 5: An illustration of the legitimate node introduced in the second method.

\section{B. Relaxation on Some Assumptions}

In our model, both CSI and the position information of eavesdroppers are assumed to be unknown to legitimate nodes. It can be seen from Figure 2 that there exists a gap between the lower bound and the upper bound of per-node capacity, when the intensity of eavesdroppers is in the range $\left[\Theta\left(n^{-\beta}\right), \Theta\left(\log \frac{\alpha-2}{\alpha} n\right)\right]$. Thus, it turns out to be a tempting issue whether the secrecy capacity can be improved if some of the information is known.

Note that the gap is caused by the randomness in Poison distribution, since a jump of $\log n$ number of nodes per unit area occurs at the point $\psi_{e}=1$. Taking the concurrent transmission range for instance, it has to be set uniform over the whole network to guarantee the transmission secrecy for each T-R pairs in the worst case. However, it can be solved in the case where the information such as the positions of eavesdroppers are known. Because different artificial noise generation powers and different concurrent transmission ranges can be exhibited at different T-R pairs. Hence, it is possible to narrow this gap, by appropriate adjustments on the number of concurrent transmission nodes as well as the corresponding TDMA schemes, which results into capacity improvement. We leave it as our future work for further investigation on the problem.

\section{Impact of Fading Gain}

Since the main purpose of this work is to investigate the secrecy capacity when both the locations of eavesdroppers and channel state information are unknown to legitimate nodes, we assume the fading gain is the same over all nodes in previous sections. And we note that when the channel state information of legitimate receiver is available to the corresponding transmitter, the main results of this paper still hold.
Intuitively, the reason behind is that the transmitter can select to transmit when the channel between legitimate T-R pair is good since the CSI is known. We consider Rayleigh fading channel here while the result can be applied to other fading channels readily. Assume the average fading gain is 1. A legitimate transmitter is scheduled to send packets only when the fading gain between the legitimate T-R pair is greater than 2. Otherwise, the transmitter will keep silent. Hence, the channel between legitimate T-R pairs is better than the channel of transmitter and eavesdroppers in the long run. And we can use the coding scheme similar to [16] to achieve the secrecy capacity. Note that the probability that the fading gain between legitimate T-R pairs is greater than 2 is a constant. Therefore, the results in our paper may suffer a constant factor and will not change in order sense.

\section{Some Extensions}

From the derivations in Section IV, it can be seen that secrecy capacity is strongly related to both the density of eavesdroppers and the distance between the T-R pairs. A larger distance between T-R pairs means that more artificial noise should be generated to decrease the eavesdroppers' SINR. Thus, the concurrent transmission range needs to be enlarged to make sure that legitimate receivers do not interfere with each other. The model presented captures the secrecy constraints by the underlying interference and relies weakly on the specific settings. Hence it can be easily extended to general networks with multicast traffic pattern or mobile nodes such as i.i.d. mobility model, one-dimensional mobility model, random walk mobility model and etc. Under the assumption that the legitimate nodes' and eavesdroppers' distribution are both Poison-distributed, the optimality of these models is preserved in secrecy concerned networks. We consider legitimate nodes do not cooperate to generate artificial noise and eavesdroppers collude to decode in the following extension. Combined with the artificial noise generation and TDMA scheduling scheme proposed in our paper, the following results are straightforward from [24], [19], [32] and [33].

Denote function $f\left(\psi_{e}(n)\right)$ as

$$
f\left(\psi_{e}(n)\right)= \begin{cases}\Theta\left(\psi_{e}(n)^{-\frac{2}{\alpha-2}}\right) & \psi_{e}(n)=\Omega\left(\log \frac{\alpha-2}{\alpha} n\right) \\ \Omega\left(\log ^{-\frac{2}{\alpha}} n\right) & \psi_{e}(n)=\left[\Omega\left(n^{-\beta}\right), O\left(\log \frac{\alpha-2}{\alpha} n\right)\right] \\ \Theta(1) & \psi_{e}(n)=O\left(n^{-\beta}\right)\end{cases}
$$

for any constant $\beta>0$.

Corollary 1. Assume that legitimate nodes and eavesdroppers are independent poisson distributed in $\mathscr{B}$ with parameter 1 and $\psi_{e}(n)$ respectively. For each legitimate node, $k-1$ nodes are randomly chosen as its destinations. For independent eavesdroppers case, the aggregated multicast secrecy capacity is $\Theta\left(\sqrt{\frac{n}{k \log n}} \cdot \log ^{-\alpha-4} n\right)$ when $k=O\left(\frac{n}{\log n}\right)$ and is $\Theta\left(\log ^{-\alpha-4} n\right)$ when $k=\Omega\left(\frac{n}{\log n}\right)$. For colluding eavesdroppers case, the aggregated multicast secrecy capacity is $\Theta\left(f\left(\psi_{e}(n)\right) \sqrt{\frac{n}{k \log n}} \cdot \log ^{-\alpha-4} n\right)$ when $k=O\left(\frac{n}{\log n}\right)$ and is $\Theta\left(f\left(\psi_{e}(n)\right) \log ^{-\alpha-4} n\right)$ when $k=\Omega\left(\frac{n}{\log n}\right)$.

Corollary 2. Consider a cell-partitioned network under the two-hop relay algorithm proposed in [19], and assume that 
nodes change cells i.i.d. and uniformly over each cell every timeslot. For independent eavesdroppers case, the per-node secrecy capacity is $\Theta(1)$ and the corresponding delay is $\Theta(n)$. For colluding case, the per-node secrecy capacity is $\Theta\left(f\left(\psi_{e}(n)\right)\right.$ and the corresponding delay is $\Theta\left(\frac{n}{f\left(\psi_{e}(n)\right)}\right)$.

Corollary 3 . Under random walk mobility model, nodes can only move to adjacent cells every timeslot. For independent eavesdroppers case, the per-node secrecy capacity is $\Theta(1)$ and the corresponding delay is $\Theta(n \log n)$. For colluding case, the per-node secrecy capacity is $\Theta\left(f\left(\psi_{e}(n)\right)\right.$ and the corresponding delay is $\Theta\left(\frac{n \log n}{f\left(\psi_{e}(n)\right)}\right)$.

Corollary 4. Since it is shown by J. Mammen and D. Shah [33] that the capacity and delay under one-dimensional random walk mobility model are the same as that without the 1-D mobility constraint which is also the case in our model. Hence, the secrecy capacity and correspondence delay are the same as that in Corollary 3.

And we note that since self-interference cancelation may be not readily applied in mobile networks, our results in mobile networks is of theoretical interest and may provide some kind of guideline. It is an interesting future work to seek more practical solutions in mobile networks.

\section{E. Comparison with Previous Work}

It is shown in [13] that the per-node secrecy throughput is $\Theta\left(\frac{1}{\sqrt{n}}\right)$ which means there is no secrecy capacity loss when $\frac{\psi_{e}}{\psi}=o\left((\log n)^{-2}\right)$. This result coincides with part of the results presented in our paper. In [13], secrecy guard zone assumed to contain no eavesdroppers existing in a region around the legitimate nodes is adopted to enhance secrecy transmission under the attack of independent eavesdroppers. However, they do not discuss in their paper how to establish a guard zone and what is the secrecy capacity when $\frac{\psi_{e}}{\psi}=\Omega\left((\log n)^{-2}\right)$.

Zhang et al. [22] show that the per-node secrecy capacity is $\Theta\left(\frac{1}{\sqrt{n \log n}}\right)$ when $p_{f}=\Omega\left(\frac{1}{\log n}\right)$ and $\Theta\left(\sqrt{\frac{p_{f}}{n}}\right)$ when $p_{f}=\left[\Omega\left(\frac{\log n}{n}\right), O\left(\frac{1}{\log n}\right)\right]$. Here $p_{f}$ represents the probability that neighboring nodes have a common key used to establish security association and is independent with the density of eavesdroppers. To guarantee the connectivity of authenticated nodes, the concurrent transmission range varies as $p_{f}$ changes. Hence, the relationship between our result and theirs is the underlying concurrent transmission chances, since we focus on the interference model whereas they rely on cryptographic techniques.

Liang et al. [16] show that the per-node secrecy capacity in mobile ad hoc network is $\Theta\left(\sqrt{\frac{D}{n}}\right)$ when $\psi_{e}=o\left(\sqrt{\frac{D}{n}}\right)$ and $\Theta\left(\frac{1}{n \psi_{e}}\right)$ when $\psi_{e}=\Omega\left(\sqrt{\frac{D}{n}}\right.$ polylog $\left.(n)\right)$, with $D$ being the delay constraint. Note that there will be chances that the closest node to the transmitter is the intended receiver when the delay is sufficiently large. Hence, the secrecy is guaranteed by the mobility of legitimate nodes while ours focus on static networks.

To make use of the channel fading gain, it can be seen that the concurrent transmission region in [12] is much larger than that in our paper. Hence more throughput has to be sacrificed to ensure the security. Furthermore, the secrecy capacity is not studied in their paper when the density of eavesdroppers is larger than 1 while we make such investigation in our work.

\section{CONCLUSION AND FUTURE WORK}

Secrecy of message delivery is a major concern in a lot of real applications. This paper studies the asymptotic behavior of secrecy capacity in an ad hoc network where both the channel state information and locations of eavesdroppers are unknown. With interference cancelation, we propose a novel construction which enhances network security greatly. Relationships of secrecy capacity and the density of eavesdroppers are investigated for both independent eavesdroppers and colluding case. Extensions to dense networks and the effect of other eavesdropping models and mobility models are also discussed. Jamming as a different kind of network attacking is also investigated. The most interesting insight in our paper perhaps is the shift on the pattern how legitimate nodes generate noises which may shed insight into the future design of wireless networks.

The secrecy issue in large-scale networks are strongly correlated with the node distributions of both legitimate nodes and eavesdroppers, also depends on how the packets is delivered across the network. Hence, it is an interesting future work to study the relationship between the secrecy capacity and the heterogeneity distributions of nodes. Also, when the network range changes or the number of legitimate nodes and eavesdroppers varies, the corresponding change in secrecy capacity is of interest. Finally, as the social network is more and more important in our daily life, how to ensure the secrecy transmission in social networks is also of great interest.

\section{ACKNOWLEDGMENT}

This paper is supported by National Fundamental Research Grant (No. 2011CB302701); NSF China (No. 61271219,61202373); Shanghai Basic Research Key Project (No. 11JC1405100); China Ministry of Education Fok Ying Tung Fund (No. 122002); China Ministry of Education New Century Excellent Talent (No. NCET-10-0580).

\section{REFERENCES}

[1] C. E. Shanon, "Communication Theorey of Secrecy Systems", in J. Bell Syst. Tech., Vol.28, pp.656-715, 1948.

[2] A. D. Wyner, "The Wire-Tap Channel", in J. Bell Sys. Tech., Vol. 54, No. 8, pp. 1355-1367, Oct. 1975.

[3] I. Csiszár and J. Körner, "Broadcast Channels with Confidential Messages", in IEEE Trans. Inform. Theory, Vol. 24, No. 3, pp. 339-348, July 1978.

[4] M. Haenggi, "The Secrecy Graph and Some of Its Properties", in Proc. IEEE ISIT, Toronto, Canada, July 2008.

[5] P. C. Pinto, J. Barros, M. Z. Win, "Wireless Secrecy in LargeScale Networks", in Proc. IEEE ITA'11, California, USA, Feb. 2011.

[6] S. Goel and R. Negi, "Guaranteeing Secrecy using Artificial Noise", in IEEE Trans. Wireless Commun., Vol. 7, No. 6, pp. 2180-2189, 2008.

[7] E. Perron, S. Diggavi, and E. Telatar, "On Cooperative Wireless Network Secrecy", in Proc. IEEE INFOCOM, Rio de Janeiro, Brazil, Apri. 2009. 
[8] T. Liu and S. Shamai, "A Note on the Secrecy Capacity of the Multiple Antenna Wiretap Channel", in IEEE Trans. Inform. Theory, Vol. 55, No. 6, pp. 2547-2553, June 2009.

[9] A. Khist and G. W. Wornell, "Secure Transmission with Multiple Antennas-Part II: The MIMOME Wiretap Channel", in IEEE Trans. Inform. Theory, Vol. 56, No. 11, pp. 5515-5532, 2010.

[10] M. Bloch, J. Barros, M. R. D. Rodrigues and S. W. McLaughlin, "Wireless Information-theoretic Security", in IEEE Trans. Inform. Theory, Vol. 54, No. 6, pp. 2515-2534, 2008.

[11] X. Wang, W. Huang, S. Wang, J. Zhang, C. Hu, "Delay and Capacity Tradeoff Analysis for MotionCast", in IEEE/ACM Trans. Networking, Vol. 19, no. 5, pp. 1354-1367, Oct 2011.

[12] S. Vasudevan, D. Goeckel and D. Towsley, "Security-capacity Trade-off in Large Wireless Networks using Keyless Secrecy", in Proc. ACM MobiHoc, Chicago, Illinois, USA, Sept. 2010.

[13] O. Koyluoglu, E. Koksal and E. Gammel, "On Secrecy Capacity Scaling in Wireless Networks", in IEEE Trans. Inform. Theory, Vol. 58, No. 5, pp. 3000-3015, 2012.

[14] X. Zhou, R. K. Ganti, J. G. Andrews and A. Hjorungnes, "The Throughput Cost of Information-Theoretic Security in Decentralized Wireless Networks", Arxiv preprint arXiv: 1012.4552.

[15] J. Zhang, L. Fu and X. Wang, "Impact of Secrecy on Capacity in Large-Scale Wireless Networks, in Proc. of IEEE INFOCOM 2012 (mini conference), Orlando, March, 2012.

[16] Y. Liang, H. V. Poor and L. Ying, "Secrecy Throughput of MANETs under Passive and Active Attacks", in IEEE Trans. Inform. Theory, Vol. 57, No. 10, pp. 6692-6702, 2011.

[17] J. I. Choiy, M. Jainy, K. Srinivasany, P. Levis and S. Katti, "Achieving Single Channel, Full Duplex Wireless Communication", in ACM Mobicom'10, Chicago, USA, Sept. 2010.

[18] P. Gupta and P. Kumar, "The Capacity of Wireless Networks", in IEEE Trans. Inform. Theory, Vol. 46, No. 2, pp. 388-404, Mar. 2000.

[19] M. J. Neely and E. Modiano, "Capacity and Delay Tradeoffs for Ad Hoc Mobile Networks", in IEEE Trans. Inform. Theory, Vol. 51, No. 6, pp. 1917-1937, 2005.

[20] M. Franceschetti, O. Dousse, D. N. Tse and P. Thiran, "Closing the Gap in the Capacity of Wireless Networks via Percolation Theory", in IEEE Trans. Inform. Theory, Vol. 53, No. 3, pp. 1009-1018, 2007.

[21] M. Garetto, P. Giaccone and E. Leonardi, "Capacity Scaling in Ad Hoc Networks with Heterogeneous Mobile Nodes: The Super-Critical Regime, in IEEE/ACM Trans. Networking, Vol. 17, No. 5, pp. 1522-1535, 2009.

[22] C. Zhang, Y. Song, Y. Fang and Y. Zhang, "On the Price of Security in Large-Scale Wireless Ad Hoc Networks", in IEEE/ACM Trans. Networking, Vol. 99, Issue 2, pp. 319-332, Apri. 2011.

[23] M. Grossglauser and D. N. C. Tse, "Mobility Increases the Capacity of Ad Hoc Wireless Networks", in IEEE/ACM Trans. Networking, Vol. 10, No. 4, pp. 477-486, 2002.

[24] X. Li, "Multicast Capacity of Wireless Ad Hoc Networks", in IEEE/ACM Trans. Networking, Vol. 17, No. 3, pp. 950-961, 2009.

[25] C. Wang, X. Li, C. Jiang, S. Tang and Y. Liu, "Multicast Throughput for Hybrid Wireless Networks under Gaussian Channel Model", in IEEE Trans. Mobile Computing, Vol. 10, No. 6, pp. 839-852, 2011.

[26] S. Tang, X. Mao, T. Jung, J. Han, X. Li, B. Xu and Chao Ma, "Closing the gap in the multicast capacity of hybrid wireless networks", in ACM MobiHoc'12, South Carolina, USA, 2012.

[27] B. Liu, P. Thiran and D. Towsley, "Capacity of a Wireless Ad Hoc Network with Infrastructure", in ACM MobiHoc'07, New York, NY, USA, 2007.

[28] A. Özgür, O. Lévêque and D.N.C. Tse, "Hierarchical Cooperation Achieves Optimal Capacity Scaling in Ad Hoc Networks", in IEEE Trans. Inform. Theory, Vol. 53, No. 10, pp. 3549-3572, 2007.
[29] M. Penrose, "Random Geometric Graphs", Oxford Univ. Press, Oxford, U.K., 2003.

[30] Xiaojun Lin, Gaurav Sharma, Ravi R. Mazumdar and Ness B. Shroff, "Degenerate Delay-Capacity Trade-offs in Ad Hoc Networks with Brownian Mobility", in the Joint Special Issue of IEEE Trans. Inform. Theory and IEEE/ACM Trans. Networking, Vol. 52, No. 6, pp. 2777-2784, 2006.

[31] T. Hagerup and C. Rüb, "A guided tour of Chernoff bounds", in Inf. Process. Lett., Vol. 33, No. 6, pp. 305-308, Feb. 1990.

[32] A. Gamal, J. Mammen, B. Prabhakar and D. Shah, "Throughput-delay trade-off in wireless networks", in Proceeding of IEEE INFOCOM, Hong Kong, China, Mar. 2004.

[33] J. Mammen and D. Shah, "Throughput and Delay in Random Wireless Networks With Restricted Mobility", in IEEE Trans. Inform. Theory, Vol. 53, No. 3, pp. 1108-1116, 2007.

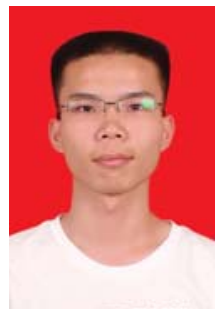

Jinbei Zhang received his B. E. degree in Electronic Engineering from Xidian University, Xi'an, China, in 2010, and is currently pursuing the Ph.D. degree in electronic engineering at Shanghai Jiao Tong University, Shanghai, China.

His current research interests include network security, capacity scaling law and mobility models in wireless networks.

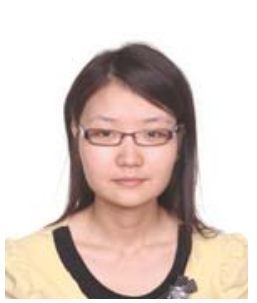

Luoyi Fu received her B. E. degree in Electronic Engineering from Shanghai Jiao Tong University, China, in 2009. She is currently working with Prof. Xinbing Wang toward the PHD degree in Department of Electronic Engineering in Shanghai Jiao Tong University.

Her research of interests are in the area of scaling laws analysis in wireless networks and performance evaluation in social networks.

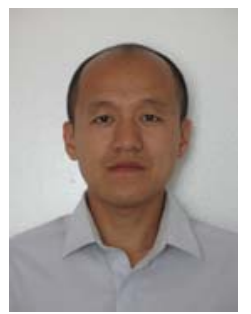

Xinbing Wang received the B.S. degree (with hons.) from the Department of Automation, Shanghai Jiaotong University, Shanghai, China, in 1998, and the M.S. degree from the Department of Computer Science and Technology, Tsinghua University, Beijing, China, in 2001. He received the Ph.D. degree, major in the Department of electrical and Computer Engineering, minor in the Department of Mathematics, North Carolina State University, Raleigh, in 2006. Currently, he is a faculty member in the Department of Electronic Engineering, Shanghai Jiaotong University, Shanghai, China. His research interests include scaling law of wireless networks and cognitive radio. Dr. Wang has been an associate editor for IEEE Transactions on Mobile Computing, and the member of the Technical Program Committees of several conferences including ACM MobiCom 2012, ACM MobiHoc 2012, IEEE INFOCOM 2009-2013. 